\title{
Global smooth solution of the Cauchy problem for a model of radiative flow
}

\author{
BERNARD DUCOMET AND ŠŚRKA NEČASOVÁ
}

\begin{abstract}
We consider a simplified model of compressible Navier-Stokes-Fourier system coupled to radiation. We prove existence and uniqueness of the solution for smooth data near a radiative equilibrium.
\end{abstract}

Mathematics Subject Classification (2010): 35Q30 (primary); 76N10 (secondary).

\section{Introduction}

We revisit a model of radiative flow investigated in [12] where the (non-relativistic) motion of the fluid is described by standard fluid mechanics giving the evolution of the mass density $\varrho=\varrho(t, x)$, the velocity field $\vec{u}=\vec{u}(t, x)$, and the absolute temperature $\vartheta=\vartheta(t, x)$ as functions of the time $t$ and the Eulerian spatial coordinate $x \in \mathbb{R}^{3}$. The effect of radiation appears through the radiative intensity $I=I(t, x, \vec{\omega}, v)$, depending on the vector $\vec{\omega} \in \mathcal{S}^{2}$, where $\mathcal{S}^{2} \subset \mathbb{R}^{3}$ denotes the unit sphere, and the frequency $v \geq 0$. The collective effect of radiation appears in the model as an average with respect to the variables $\vec{\omega}$ and $v$ of a quantity depending on $I$ : the radiation energy $E^{R}$ given by

$$
E^{R}(t, x)=\frac{1}{c} \int_{\mathcal{S}^{2}} \int_{0}^{\infty} I(t, x, \vec{\omega}, v) \mathrm{d} \vec{\omega} \mathrm{d} v
$$

and the radiation momentum $\vec{F}^{R}$, given by

$$
\vec{F}^{R}(t, x)=\int_{\mathcal{S}^{2}} \int_{0}^{\infty} \vec{\omega} I(t, x, \vec{\omega}, v) \mathrm{d} \vec{\omega} \mathrm{d} v .
$$

The time evolution of $I$ itself is described by a transport equation with a source term depending on the absolute temperature, while the effect of radiation on the

Š. N. was supported by the Czech Agency of the Czech Republic n.201/08/0012 and by RVO 67985840.

Received August 1, 2012; accepted in revised version November 7, 2012. 
macroscopic motion of the fluid is represented by an extra source term in the energy equation evaluated in terms of $I$. More specifically, the system of equations to be studied reads as follows:

$$
\begin{gathered}
\partial_{t} \varrho+\operatorname{div}_{x}(\varrho \vec{u})=0 \text { in }(0, T) \times \mathbb{R}^{3}, \\
\partial_{t}(\varrho \vec{u})+\operatorname{div}_{x}(\varrho \vec{u} \otimes \vec{u})+\nabla_{x} p=\operatorname{div}_{x} \mathbb{S}-\vec{S}_{F} \text { in }(0, T) \times \mathbb{R}^{3}, \\
\partial_{t}(\varrho e)+\operatorname{div}_{x}(\varrho e \vec{u})+\operatorname{div}_{x} \vec{q}=\mathbb{S}: \nabla_{x} \vec{u}-p \operatorname{div}_{x} \vec{u}-S_{E} \text { in }(0, T) \times \mathbb{R}^{3}, \\
\frac{1}{c} \partial_{t} I+\vec{\omega} \cdot \nabla_{x} I=S \text { in }(0, T) \times \mathbb{R}^{3} \times(0, \infty) \times \mathcal{S}^{2} .
\end{gathered}
$$

In the right-hand sides of (1.4) and (1.5) there appear the (radiative) momentum source

$$
\vec{S}_{F}(t, x)=\int_{\mathcal{S}^{2}} \int_{0}^{\infty} \vec{\omega} \cdot S(t, x, \vec{\omega}, v) \mathrm{d} \vec{\omega} \mathrm{d} v,
$$

and the (radiative) energy source

$$
S_{E}(t, x)=\int_{\mathcal{S}^{2}} \int_{0}^{\infty} S(t, x, \vec{\omega}, v) \mathrm{d} \vec{\omega} \mathrm{d} v
$$

then, integrating (1.6) on $(0, T) \times \mathbb{R}^{3} \times(0, \infty) \times \mathcal{S}^{2}$, one observes that (1.5) and (1.6) imply the total energy conservation

$$
\begin{aligned}
\partial_{t}\left(\varrho\left(\frac{1}{2}|\vec{u}|^{2}+e\right)+E^{R}\right) & +\operatorname{div}_{x}\left(\varrho\left(\frac{1}{2}|\vec{u}|^{2}+e\right) \vec{u}+\vec{F}^{R}\right) \\
& +\operatorname{div}_{x}(p \vec{u}+\vec{q}-\mathbb{S} \vec{u}) \\
& =-\vec{u} \cdot \vec{S}_{F} \text { in }(0, T) \times \mathbb{R}^{3} .
\end{aligned}
$$

The symbol $p=p(\varrho, \vartheta)$ denotes the thermodynamic pressure and $e=e(\varrho, \vartheta)$ is the specific internal energy.

The viscous stress tensor $\mathbb{S}$ is determined by

$$
\mathbb{S}=2 \mu \mathbb{D}+\lambda \operatorname{div}_{x} \vec{u} \mathbb{I},
$$

where $\mathbb{D} \equiv\left(\nabla_{x} \vec{u}+\nabla_{x}^{t} \vec{u}\right)$ is the strain tensor and $\mu(\varrho, \vartheta)>0$ and $\lambda(\varrho, \vartheta) \geq 0$ are the viscosity coefficients. The right-hand side of (1.4) can be rewritten

$$
\operatorname{div}_{x} \mathbb{S}=\mu \Delta \vec{u}+(\lambda+\mu) \nabla_{x}\left(\operatorname{div}_{x} \vec{u}\right)+2 \nabla_{x} \mu \cdot \mathbb{D}+\nabla_{x} \lambda \operatorname{div}_{x} \vec{u} .
$$

The heat flux $\vec{q}$ is given by the Fourier's law

$$
\vec{q}=-\kappa \nabla_{x} \vartheta,
$$

with the heat conductivity coefficient $\kappa(\varrho, \vartheta)>0$. 
Finally,

$$
S=S_{a, e}+S_{s}
$$

where

$$
S_{a, e}=\sigma_{a}(B(\nu, \vartheta)-I), S_{s}=\sigma_{s}\left(\frac{1}{4 \pi} \int_{\mathcal{S}^{2}} I(\cdot, \vec{\omega}) \mathrm{d} \vec{\omega}-I\right)
$$

with $B(v, \vartheta)=2 h v^{3} c^{-2}\left(e^{\frac{h v}{k \vartheta}}-1\right)^{-1}$ the radiative equilibrium function where $h$ and $k$ are the Planck and Boltzmann constants, $\sigma_{a}=\sigma_{a}(\nu, \vartheta) \geq 0$ is the absorption coefficient and $\sigma_{s}=\sigma_{s}(v, \vartheta) \geq 0$ is the scattering coefficient. More restrictions on these structural properties of constitutive quantities will be imposed in Section 2 below.

System $(1.3-1.6)$ is supplemented with the initial conditions

$$
\begin{gathered}
(\varrho, \vec{u}, \vartheta)(0, x)=\left(\varrho_{0}, \vec{u}_{0}, \vartheta_{0}\right)(x), \quad x \in \mathbb{R}^{3}, \\
I(0, x, v, \vec{\omega})=I_{0}(x, v, \vec{\omega}) \text { for } x \in \mathbb{R}^{3}, \vec{\omega} \in \mathcal{S}^{2}, v \in \mathbb{R}_{+} .
\end{gathered}
$$

System (1.3 - 1.14) can be viewed as a simplied model in radiation hydrodynamics, the physical foundations of which were described by Pomraning [42] and Mihalas and Weibel-Mihalas [40] in the full framework of special relativity. Similar systems have been investigated more recently in astrophysics by Lowrie, Morel and Hittinger [36], Buet and Després [6], with a special attention to asymptotic regimes, see also Dubroca and Feugeas [10], Lin [33] and Lin, Coulombel and Goudon [34] for related numerical issues. Recall that a simplified version of the system (non conducting fluid at rest) has also been investigated by Golse and Perthame [22] (see also [23,24]), where global existence was proved by means of the theory of nonlinear semi-groups under very general hypotheses on the transport coefficients.

Let us mention that a global existence result has been proved recently in the 3D setting in [12] for large data under some cut-off hypotheses on transport coefficients, a global existence result has also been proved in the stationary case in [31] for large data under different cut-off hypotheses on transport coefficient and that the low Mach number regime for a system close to (1.3 - 1.14) introduced by Seaïd, Teleaga and col. in $[11,46,47]$, has also been investigated in [17]. Moreover a number of similar results concerning global existence and asymptotic behaviour for large times have also been considered in the recent past in the one-dimensional geometry $[1,13-16]$. Finally in the compressible but inviscid setting, the existence of a localin-time solution has been proved recently in [48] together with the existence of global weak solutions to the Euler-Boltzmann equations in radiation hydrodynamics in [30] and the Cauchy problem for an inviscid diffusion model was investigated in [35]. 
Our goal in the following is to prove the global existence of a strong solution to $(1.3-1.14)$. As far as a strong solution is concerned, we need to restrict ourselves to well prepared data in a neighbourhood of an equilibrium. A number of authors deal with global solutions of the perturbative compressible heat-conducting NavierStokes system, starting with the pioneer article by J. Nash [41]. Among these, let us quote the works of N. Itaya [28], A. Matsumura and T. Nishida [37,38], Bui An Ton [7], and recently D. Hoff [25-27] and S. Jiang [29].

In the following, we consider the global existence of a solution of the Cauchy problem for the system (1.3)-(1.6) near a (radiative) equilibrium, using the methods introduced by Hoff in [25-27], extending a previous work of S. Jiang [29] who considered a perfect gas without radiation.

The paper is organized as follows. In Section 2, we list the principal hypotheses imposed on constitutive relations and state the main result. In Section 3 we derive necessary $L^{p}$-estimates for the unknowns (density, velocity, temperature and radiative energy). In Section 4 we give the proof of Proposition 2.3 and in Section 5 we give the proof of Theorem 2.2. Finally in the Appendix is given, for completeness, a short proof of local existence of a solution for our Cauchy problem.

ACKnowledgements. Part of the paper was written during the second author's stay in CEA. She would like to thank to Prof. Ducomet for his hospitality during her stay.

\section{Hypotheses and main results}

We suppose that the state functions (pressure $p=p(\varrho, \vartheta)$ and internal energy $e=e(\varrho, \vartheta)$ ) are smooth positive functions of their arguments submitted to the thermodynamical constraint $\frac{\partial e}{\partial \vartheta}>0$ and related through Maxwell's equation

$$
\frac{\partial e}{\partial \varrho}=\frac{1}{\varrho^{2}}\left(p(\varrho, \vartheta)-\vartheta \frac{\partial p}{\partial \vartheta}\right)
$$

In order to simplify the arguments, we suppose in the sequel that the transport coefficients $\mu, \eta$, and $\kappa$ are positive constants. Moreover we assume that $\sigma_{a}, \sigma_{s}, B$ are continuous functions of $\nu, \vartheta$ such that

$$
\begin{gathered}
0 \leq \sigma_{a}(\nu, \vartheta), \sigma_{s}(\nu, \vartheta),\left|\partial_{\vartheta} \sigma_{a}(\nu, \vartheta)\right|,\left|\partial_{\vartheta} \sigma_{a}(\nu, \vartheta)\right| \leq c_{1}, \\
0 \leq \sigma_{a}(\nu, \vartheta) B(\nu, \vartheta),\left|\partial_{\vartheta}\left\{\sigma_{a}(\nu, \vartheta) B(\nu, \vartheta)\right\}\right| \leq c_{2}, \\
\sigma_{a}(\nu, \vartheta), \sigma_{s}(\nu, \vartheta), \sigma_{a}(\nu, \vartheta) B(\nu, \vartheta) \leq h(v), h \in L^{1}(0, \infty),
\end{gathered}
$$

for all $v \geq 0, \vartheta \geq 0$. Relations (2.2 - 2.4) represent "cut-off" hypotheses neglecting the effect of radiation at large frequencies $v$.

It will be convenient to replace (1.5) by the internal energy equation

$$
\partial_{t}(\varrho e)+\operatorname{div}_{x}(\varrho e \vec{u})+\operatorname{div}_{x} \vec{q}=\mathbb{S}: \nabla_{x} \vec{u}-p \operatorname{div}_{x} \vec{u}-S_{E} .
$$


Furthermore, dividing (2.5) by $\vartheta$ and using Maxwell's relation (2.1), we get the entropy equation

$$
\partial_{t}(\varrho s)+\operatorname{div}_{x}(\varrho s \vec{u})+\operatorname{div}_{x}\left(\frac{\vec{q}}{\vartheta}\right)=\varsigma,
$$

where

$$
\varsigma=\frac{1}{\vartheta}\left(\mathbb{S}: \nabla_{x} \vec{u}-\frac{\vec{q} \cdot \nabla_{x} \vartheta}{\vartheta}\right)-\frac{S_{E}}{\vartheta}+\frac{\vec{u} \cdot \vec{S}_{F}}{\vartheta},
$$

where the first term $\varsigma_{m}:=\frac{1}{\vartheta}\left(\mathbb{S}: \nabla_{x} \vec{u}-\frac{\vec{q} \cdot \nabla_{x} \vartheta}{\vartheta}\right)$ is the (positive) matter entropy production. In order to identify the second term we recall [2] the formula for the entropy of a photon gas

$$
s^{R}=-\frac{2 k}{c^{3}} \int_{0}^{\infty} \int_{\mathcal{S}^{2}} v^{2}[n \log n-(n+1) \log (n+1)] d \vec{\omega} d v,
$$

where $n=n(I)=\frac{c^{2} I}{2 h v^{3}}$ is the occupation number. Defining the radiative entropy flux

$$
\vec{q}^{R}=-\frac{2 k}{c^{2}} \int_{0}^{\infty} \int_{\mathcal{S}^{2}} \nu^{2}[n \log n-(n+1) \log (n+1)] \vec{\omega} d \vec{\omega} d \nu
$$

and using the radiative transfer equation, we get the equation

$$
\partial_{t} s^{R}+\operatorname{div}_{x} \vec{q}^{R}=-\frac{k}{h} \int_{0}^{\infty} \int_{\mathcal{S}^{2}} \frac{1}{v} \log \frac{n}{n+1} S d \vec{\omega} d v=: \varsigma^{R} .
$$

Checking the identity $\log \frac{n(B)}{n(B)+1}=\frac{h v}{k \vartheta}$ with $B=B(\vartheta, v)$ the Planck function, and using the definition of $S$, the right-hand side of (2.10) rewrites as

$$
\begin{aligned}
\varsigma^{R}= & \frac{S_{E}}{\vartheta}-\frac{\vec{u} \cdot \vec{S}_{F}}{\vartheta} \\
& -\frac{k}{h} \int_{0}^{\infty} \int_{\mathcal{S}^{2}} \frac{1}{v}\left[\log \frac{n(I)}{n(I)+1}-\log \frac{n(B)}{n(B)+1}\right] \sigma_{a}(B-I) d \vec{\omega} d v \\
& -\frac{k}{h} \int_{0}^{\infty} \int_{\mathcal{S}^{2}} \frac{1}{v}\left[\log \frac{n(I)}{n(I)+1}-\log \frac{n(\tilde{I})}{n(\tilde{I})+1}\right] \sigma_{S}(\tilde{I}-I) d \vec{\omega} d v
\end{aligned}
$$

where we used the hypothesis that the transport coefficients $\sigma_{a, s}$ do not depend on $\vec{\omega}$. So we finally obtain

$$
\partial_{t}\left(\varrho s+s^{R}\right)+\operatorname{div}_{x}\left(\varrho s \vec{u}+\vec{q}^{R}\right)+\operatorname{div}_{x}\left(\frac{\vec{q}}{\vartheta}\right)=\varsigma^{m}+\varsigma^{R} .
$$

Let us observe after [6] that only the first two terms in the right-hand side of (2.11) have a definite sign, satisfying the positivity of entropy production rate and that 
the last integral involving $\vec{S}_{F}$ is only positive up to a relativistic $\frac{\vec{u}}{c}$ correction. This fact exhibit a lack of consistency of a model coupling a non-relativistic fluid to a relativistic photon flow.

Let us give now some definitions used throughout the paper. For any second rank tensor (matrix) we write the complete contraction as $M: M \equiv \sum_{i, k=1}^{3} M_{i k} M_{i k}$.

We denote $L^{p}:=L^{p}\left(\mathbb{R}^{3}\right)\left(\right.$ resp. $W^{m, p}:=W^{m, p}\left(\mathbb{R}^{3}\right)$ ) the standard Lebesgue (resp. Sobolev) spaces for $p \geq 1$, with norms $\|\cdot\|_{L^{p}}$ (resp. $\|\cdot\|_{W^{m, p}}$ ). For a function depending on space, angular variable and frequency, we will also note $L^{p}$ for $L^{p}\left(\mathbb{R}^{3} \times \mathcal{S}^{2} \times \mathbb{R}_{+}\right)$. We write $\|\cdot\|$ for $\|\cdot\|_{L^{2}}, H^{m}$ for $W^{m, 2}$ and $\int f d x$ for $\int_{\mathbb{R}^{3}} f(x) d x$.

We denote the vorticity tensor by $\omega=\frac{1}{2}\left(\nabla_{x} \vec{u}-\nabla_{x}^{t} \vec{u}\right)$, with components

$$
\omega_{i j}:=\frac{1}{2}\left(\partial_{i} u_{j}-\partial_{j} u_{i}\right)
$$

The Lagrangian derivative of a quantity $f$ is given by

$$
\dot{f} \equiv \frac{d}{d t} f:=\partial_{t} f+\vec{u} \cdot \nabla_{x} f .
$$

Moreover one has the commutation rule

$$
\frac{d}{d t} \int \varrho \Phi d x=\int \varrho \dot{\Phi} d x
$$

Recall the following Euler-Lagrange result (see [25, Lemma 3.2]).

Lemma 2.1. Let $t_{0} \in[0, T]$ be fixed. We define the family of particle trajectories $x(y, t)$ by

$$
\frac{d x}{d t}(y, t)=\vec{u}(x(y, t), t), \quad x\left(y, t_{0}\right)=y .
$$

Let $g \in L^{1}(0, T), g \geq 0$, and let $t \in[0, T]$. Then each of the integrals

$$
\int g(x(y, t), t) d y \text { and } \int g(x, t) d x
$$

is bounded by $C$ times the other, where $C$ is a positive constant.

The effective viscous pressure $F$ is generally defined by (see [19], and also $[25,26]$ in the constant coefficients case)

$$
F(t):=\left(\nabla_{x} \Delta^{-1} \nabla_{x}\right): \mathbb{S}-(p-\bar{p}) .
$$

It is well defined provided $\mathbb{S} \in L^{2}\left(0, T ; L^{2}\left(\mathbb{R}^{3} ; \mathbb{R}^{9}\right)\right.$ ) (see [19]) and the singular operator rewrites as

$$
\left(\nabla_{x} \Delta^{-1} \nabla_{x}\right)_{i, j} \equiv-\mathcal{R}_{i} \mathcal{R}_{j}
$$


where $\mathcal{R}_{i}$ is the Riesz operator with $\operatorname{symbol} \mathcal{R}_{i}(\xi)=\frac{i \xi_{i}}{|\xi|}$. Recall [19] that as $\lambda$ and $\mu$ are constant

$$
\begin{aligned}
& \left(\nabla_{x} \Delta^{-1} \nabla_{x}\right): \mathbb{S} \\
& =\mathcal{F}_{\xi \rightarrow x}^{-1}\left[i \frac{\xi_{i} \xi_{j}}{|\xi|^{2}}\left(\mu\left(\xi_{j} \mathcal{F}_{x \rightarrow \xi}\left[u_{i}\right]+\xi_{i} \mathcal{F}_{x \rightarrow \xi}\left[u_{j}\right]\right)+\lambda \delta_{i, j} \xi_{k} \mathcal{F}_{x \rightarrow \xi}\left[u_{k}\right]\right)\right] \\
& \equiv(2 \mu+\lambda) \operatorname{div}_{x} \vec{u},
\end{aligned}
$$

and we get the simple formula

$$
F(t):=(2 \mu+\lambda) \operatorname{div}_{x} \vec{u}-(p-\bar{p}) .
$$

For an equilibrium state defined by $(\bar{\varrho}, 0, \bar{\vartheta}, \bar{I})$, with $\bar{\varrho}>0, \bar{\vartheta}>0$ and $\bar{I}>0$ with the compatibility condition $\bar{I}=B(v, \bar{\vartheta})$, we put

$$
\begin{aligned}
e_{0}:= & \left\|\varrho_{0}-\bar{\varrho}\right\|+\left\|\varrho_{0}-\bar{\varrho}\right\|_{L^{\infty}}+\left\|\vec{u}_{0}\right\|_{H^{1}}+\left\|\vartheta_{0}-\bar{\vartheta}\right\|_{H^{1}} \\
& +\left\|E_{0}^{R}-\overline{E^{R}}\right\|+\left\|\vec{U}_{0}\right\|+\left\|\omega_{0}\right\|_{L^{4}}^{2},
\end{aligned}
$$

and

$$
E_{0}:=e_{0}+\left\|\nabla_{x} \varrho_{0}\right\|+\left\|\nabla_{x} \varrho_{0}\right\|_{L^{\alpha}}+\left\|\nabla_{x} I_{0}\right\|+\left\|\nabla_{x} I_{0}\right\|_{L^{\alpha}}+\left\|\nabla_{x} \vec{U}_{0}\right\|,
$$

for $3<\alpha<6$, with

$$
\begin{gathered}
\vec{U}_{0}:=\varrho_{0}^{-1}\left(\mu \Delta \vec{u}_{0}+(\lambda+\mu) \nabla_{x} \operatorname{div}_{x} \vec{u}_{0}-\nabla_{x} p\left(\varrho_{0}, \vartheta_{0}\right)\right), \\
\omega_{0}:=\left.\omega_{0}\right|_{t=0},
\end{gathered}
$$

and

$$
\overline{E^{R}}=\int_{0}^{\infty} B(v, \bar{\vartheta}) d v .
$$

Finally for a $T>0$, we measure the size of the solution by the quantity

$$
\begin{aligned}
A(T):= & \sup _{t \in[0, T]}\left\{\|\varrho-\bar{\varrho}\|^{2}+\|\vartheta-\bar{\vartheta}\|^{2}+\|\vec{u}\|^{2}+\left\|\nabla_{x} \vec{u}\right\|^{2}+\left\|\nabla_{x} \vartheta\right\|^{2}\right. \\
& \left.+\left\|E^{R}-\overline{E^{R}}\right\|^{2}+\|\dot{\vec{u}}\|^{2}+\|\omega\|_{L^{4}}^{4}+\phi^{2}\|\Delta \vartheta\|^{2}+\phi\|\dot{\vartheta}\|^{2}\right\}(t) \\
& +\int_{0}^{T}\left\{\left\|\nabla_{x} \vec{u}\right\|^{2}+\left\|\nabla_{x} \vartheta\right\|^{2}+\left\|\nabla_{x} \omega\right\|^{2}+\|\dot{\vec{u}}\|^{2}+\|\dot{\vartheta}\|^{2}+\left\|\dot{E^{R}}\right\|^{2}\right. \\
& \left.+\|\Delta \vartheta\|^{2}+\left\|\nabla_{x} \vec{u}\right\|_{L^{4}}^{4}+\left\|\nabla_{x} \dot{\vec{u}}\right\|^{2}+\phi\left\|\nabla_{x} \dot{\vartheta}\right\|^{2}+\left\|\nabla_{x} I\right\|^{2}\right\}(t) d t,
\end{aligned}
$$

where $\phi \equiv \phi(t):=\min \{t, 1\}$. 
The main result of the present paper can be stated as follows:

Theorem 2.2. Let $\left(\varrho_{0}-\bar{\varrho}, \vec{u}_{0}, \vartheta_{0}-\bar{\vartheta}, E_{0}^{R}-\overline{E^{R}}\right) \in H^{3}\left(\mathbb{R}^{3}\right)$ with inf $\vartheta_{0}>0$. Then there exists a positive constant $\varepsilon_{1} \leq 1$ depending only on the physical constants such that if $E_{0} \leq \varepsilon_{1}$, the Cauchy problem (1.3 - 1.6), has a global unique solution $(\varrho, \vec{u}, \vartheta, I)$ satisfying

$$
\begin{gathered}
\left(\varrho-\bar{\varrho}, \vec{u}, \vartheta-\bar{\vartheta}, E^{R}-\overline{E^{R}}\right) \in C\left([0, \infty), H^{3}\left(\mathbb{R}^{3}\right)\right), \\
\partial_{t} \vec{u}, \partial_{t} \vartheta \in C\left([0, \infty), H^{1}\left(\mathbb{R}^{3}\right)\right) \cap L^{2}\left([0, \infty), H^{2}\left(\mathbb{R}^{3}\right)\right), \\
\partial_{t} \varrho \in C\left([0, \infty), H^{2}\left(\mathbb{R}^{3}\right)\right) \cap L^{2}\left([0, \infty), H^{2}\left(\mathbb{R}^{3}\right)\right), \\
\sup _{t \geq 0}\|\varrho-\bar{\varrho}\|_{L^{\infty}} \leq \frac{1}{2} \bar{\varrho}, \sup _{t \geq 0}\left\|E^{R}-\overline{E^{R}}\right\|_{L^{\infty}} \leq \frac{1}{2} \overline{E^{R}}, \inf _{\mathbb{R}^{3}, \mathbb{R}_{+}} \vartheta(x, t)>0 .
\end{gathered}
$$

Using the same strategies as in [25] and [29], the proof of Theorem 2.2 will be achieved provided that we prove a local existence result for the Cauchy problem (1.3 - 1.6), on a small interval $\left[0, T_{*}\right]$, which will be obtained using a fixed point theorem (see the Appendix), and provided we prove suitable global estimates allowing to continuate the solution beyond $T_{*}$.

These a-priori estimates are the matter of the following proposition:

Proposition 2.3. Let $(\varrho, \vec{u}, \vartheta, I)$ be a smooth solution of (1.3-1.6), defined for $t \in[0, T]$. Then there exist positive constants $\varepsilon \leq 1$ and $K$ depending only on the physical constants such that if $e_{0} \leq \varepsilon$ then

$$
\begin{aligned}
A(T) & \leq K e_{0}^{2}, \quad \sup _{t \in[0, T]}\left\{\|\varrho-\bar{\varrho}\|_{L^{\infty}}+\phi(t)\|\vartheta-\bar{\vartheta}\|_{L^{\infty}}+\left\|E^{R}-\overline{E^{R}}\right\|_{L^{\infty}}\right\} \\
& \leq K e_{0},
\end{aligned}
$$

and for all $q \in(2, \infty)$

$$
\left\{\|\varrho(T)-\bar{\varrho}\|_{L^{\infty}}+\|\vartheta(T)-\bar{\vartheta}\|_{L^{q}}+\|\vec{u}(T)\|_{L^{q}}+\left\|E^{R}(T)-\overline{E^{R}}\right\|_{L^{\infty}}\right\} \rightarrow 0
$$

as $T \rightarrow \infty$.

Our main goal in the following will be to prove Proposition 2.3. As our system is a perturbation of the viscous compressible Navier-Stokes system studied by S. Jiang in [29], we essentially follow his strategy (see also the closely related works of D. Hoff [25,26] and [27]) and insist only on the difficulties involved in the radiative coupling. As previously evoked, once we have shown Proposition 2.3, the 
proof of Theorem 2.2 will follow the lines of the proof in [29, Theorem 1.2] so we will only sketch its proof in the last section.

\section{A priori estimates on radiative and hydrodynamic variables}

Let $e_{0} \leq 1$ and assume that $(\varrho, \vartheta, I)$ satisfy

$$
|\varrho(x, t)-\bar{\varrho}|<C, \quad \phi(t)|\vartheta-\bar{\vartheta}|<C, \quad\left|E^{R}-\overline{E^{R}}\right|<C
$$

where $C \leq \frac{1}{2} \min \left\{\bar{\varrho}, \bar{\vartheta}, \overline{E^{R}}\right\}$, for all $x \in \mathbb{R}^{3}, t \in(0, T)$.

\section{1. $L^{p}$-estimates for density}

We will show in this section that $\sup _{t \geq 0}\|\varrho-\bar{\varrho}\|_{L^{\infty}}$ and $\int_{0}^{T}\|\varrho-\bar{\varrho}\|_{L^{4}}^{4} d t$ can be bounded by suitable powers of $e_{0}$ and $\bar{A}$.

Lemma 3.1. Let $1<p<\infty$. The following estimates hold for the velocity $\vec{u}$ and the effective viscous flux $F$

$$
\begin{gathered}
\left\|\nabla_{x} \vec{u}\right\|_{L^{p}} \leq C\left(\|F\|_{L^{p}}+\|\omega\|_{L^{p}}+\|\varrho-\bar{\varrho}\|_{L^{p}}+\|\vartheta-\bar{\vartheta}\|_{L^{p}}+\|I-\bar{I}\|_{L^{p}}\right), \\
\left\|\nabla_{x} F\right\|_{L^{p}} \leq C\left(\|\dot{\vec{u}}\|_{L^{p}}+\|\vartheta-\bar{\vartheta}\|_{L^{p}}+\|I-\bar{I}\|_{L^{p}}\right) \\
\int_{0}^{t}\|F\|_{L^{4}} d s \leq C A^{2}(T), \\
\int_{0}^{t}\|F\|_{L^{\infty}} d s \leq C A^{1 / 2}(T), \\
\int_{0}^{t}\left\|\nabla_{x} \vec{u}: \nabla_{x} \vec{u}\right\|^{2} d s \leq C\left(e_{0}^{2}+A^{2}(T)+\int_{0}^{t}\|\omega: \omega\|^{2} d s\right) .
\end{gathered}
$$

Proof.

1. Applying the divergence operator to (1.4) we get after (2.16)

$$
\Delta p=\operatorname{div}_{x} \operatorname{div}_{x} \mathbb{S}-\operatorname{div}_{x} \varrho \dot{\vec{u}}-\operatorname{div}_{x} \vec{S}_{F}
$$

Then

$$
\Delta F=\Delta\left(\nabla_{x} \Delta^{-1} \nabla_{x}: \mathbb{S}-(p-\bar{p})\right)=\operatorname{div}_{x} \varrho \dot{\vec{u}}-\operatorname{div}_{x} \vec{S}_{F}
$$


As $\bar{I}$ and the radiative coefficients are independent of $\omega$, we have

$$
\begin{aligned}
\vec{S}_{F}(\vartheta, I)= & \vec{S}_{F}(\vartheta, I)-\vec{S}_{F}(\bar{\vartheta}, \bar{I})=\int_{\mathcal{S}^{2}} \int_{0}^{\infty} \vec{\omega}[S(t, x, \vec{\omega}, v)-\bar{S}] \mathrm{d} \vec{\omega} \mathrm{d} v \\
= & \int_{\mathcal{S}^{2}} \int_{0}^{\infty} c \vec{\omega}[\Sigma(\nu, \vartheta) I(t, x, \vec{\omega}, v)-\Sigma(v, \bar{\vartheta}) \bar{I}] \mathrm{d} \vec{\omega} \mathrm{d} v \\
& +\int_{\mathcal{S}^{2}} \int_{0}^{\infty} c \vec{\omega}\left[\sigma_{a}(B-\bar{B})+\sigma_{S}(\tilde{I}-\bar{I})\right] \mathrm{d} \vec{\omega} \mathrm{d} \nu
\end{aligned}
$$

with $\Sigma(\nu, \vartheta)=\sigma_{a}(v, \vartheta)+\sigma_{s}(\nu, \vartheta)$. Taking Fourier transform implies, after (2.17)

$$
\partial_{j} \mathcal{F}_{x \rightarrow \xi}[F]=\frac{\xi_{i} \xi_{j}}{|\xi|^{2}} \mathcal{F}_{x \rightarrow \xi}[\varrho \dot{\vec{u}}]-\frac{\xi_{i} \xi_{j}}{|\xi|^{2}} \mathcal{F}_{x \rightarrow \xi}\left[\vec{S}_{F}-\overline{\vec{S}_{F}}\right]
$$

Using the Hörmander-Mikhlin theorem (see [21,45]) with Fourier multiplier $m(\xi):=\frac{\xi_{i} \xi_{j}}{|\xi|^{2}}$, we find (3.3).

2. Estimate (3.2) is proved in the same stroke by introducing the vorticity and the definition of $F$ in (1.4). In fact checking the identity

$$
(\lambda+2 \mu) \Delta \vec{u}=\nabla_{x} F+(\lambda+2 \mu) \operatorname{div}_{x} \omega+\nabla_{x}(p-\bar{p})-\vec{S}_{F}+\overline{\vec{S}}_{F},
$$

observing that, using a Taylor expansion, $p-\bar{p}$ rewrites

$$
\begin{aligned}
p(\varrho, \vartheta)-p(\bar{\varrho}, \bar{\varrho})= & \int_{0}^{1}\left[(\varrho-\bar{\varrho}) p_{\varrho}(\bar{\varrho}+s(\varrho-\bar{\varrho}), \bar{\vartheta})\right. \\
& \left.+(\vartheta-\bar{\vartheta}) p_{\vartheta}(\bar{\varrho}, \bar{\vartheta}+s(\vartheta-\bar{\vartheta}))\right] d s
\end{aligned}
$$

and taking Fourier transform in (3.8), we get

$$
\begin{aligned}
(\lambda+2 \mu) \mathcal{F}_{x \rightarrow \xi}\left[\partial_{i} u_{j}\right]= & \frac{\xi_{i} \xi_{j}}{|\xi|^{2}} \mathcal{F}_{x \rightarrow \xi}[F]+(\lambda+2 \mu) \frac{\xi_{k} \xi_{i}}{|\xi|^{2}} \mathcal{F}_{x \rightarrow \xi}\left[\omega_{j k}\right] \\
& +\frac{\xi_{i} \xi_{j}}{|\xi|^{2}} \mathcal{F}_{x \rightarrow \xi}[p-\bar{p}]-\frac{\xi_{i} \xi_{j}}{|\xi|^{2}} \mathcal{F}_{x \rightarrow \xi}\left[\vec{S}_{F}-\vec{S}_{F}\right]
\end{aligned}
$$

Applying once more the Hörmander-Mikhlin theorem with the same Fourier multiplier $m(\xi) \frac{\xi_{i} \xi_{j}}{|\xi|^{2}}$, we find (3.2).

3. Estimates (3.4) and (3.5) follow from the definition of $A(T)$. 
4. Estimate (3.6) is a consequence of (3.2) and (3.3).

Then we can estimate $\int_{0}^{T}\|\varrho-\bar{\varrho}\|_{L^{4}}^{4} d t$.

\section{Lemma 3.2 .}

1. $\sup _{t \geq 0}\|\varrho-\bar{\varrho}\|_{L^{4}}^{4}+\int_{0}^{T}\|\varrho-\bar{\varrho}\|_{L^{4}}^{4} d t \leq C\left(e_{0}^{4}+A^{2}(T)\right)$,

2.

$$
\sup _{t \geq 0}\|\varrho-\bar{\varrho}\|_{L^{\infty}} \leq C\left(e_{0}+A^{1 / 2}(T)\right) .
$$

Proof.

1. Using the definition of $F$, we rewrite (1.3) as

$$
(\lambda+2 \mu) \frac{d}{d t}(\varrho-\bar{\varrho})=-\varrho F-\varrho(p-\bar{p}),
$$

and multiplying by $(\varrho-\bar{\varrho})^{3}$,we get

$$
\frac{1}{4}(\lambda+2 \mu) \frac{d}{d t}(\varrho-\bar{\varrho})^{4}=-\varrho(\varrho-\bar{\varrho})^{3} F-\varrho(p-\bar{p})(\varrho-\bar{\varrho})^{3} .
$$

Using the previous decomposition

$$
\begin{aligned}
p(\varrho, \vartheta)-p(\bar{\varrho}, \bar{\vartheta})= & (\varrho-\bar{\varrho}) \int_{0}^{1} \partial_{\varrho} p(\bar{\varrho}+s(\varrho-\bar{\varrho}), \vartheta) d s \\
& +(\vartheta-\bar{\vartheta}) \int_{0}^{1} \partial_{\vartheta} p(\bar{\varrho}, \bar{\vartheta}+s(\vartheta-\bar{\vartheta})) d s,
\end{aligned}
$$

and observing that, by (3.1) and thermodynamical stability

$$
\left\{\begin{array}{l}
\partial_{\varrho} p(\bar{\varrho}+s(\varrho-\bar{\varrho}), \vartheta) \geq C_{1}(\bar{\varrho}, \bar{\vartheta}), \\
\left|\partial_{\vartheta} p(\bar{\varrho}, \bar{\vartheta}+s(\vartheta-\bar{\vartheta}))\right| \leq C_{2}(\bar{\varrho}, \bar{\vartheta}),
\end{array}\right.
$$

for two positive constants $C_{1}, C_{2}$. We get after (3.1)

$$
\begin{aligned}
\frac{1}{4}(\lambda+2 \mu) \frac{d}{d t}(\varrho-\bar{\varrho})^{4}+C_{1} \frac{\bar{\varrho}}{2}(\varrho-\bar{\varrho})^{4} \leq & \frac{\varepsilon}{4}(\varrho-\bar{\varrho})^{4} \\
& +C_{\varepsilon} F^{4}+\frac{\varepsilon}{4}(\varrho-\bar{\varrho})^{4}+C_{\varepsilon}(\vartheta-\bar{\vartheta})^{4} .
\end{aligned}
$$


Choosing $\varepsilon<\frac{C_{1} \bar{Q}}{2}$, integrating on a fixed particle trajectory $x(y, t)$ and using Lemma 2.1, we get

$$
\begin{aligned}
& (\varrho-\bar{\varrho})^{4}(x(y, t), t)+\frac{2 C_{1} \bar{\varrho}}{\lambda+2 \mu} \int_{0}^{t}(\varrho-\bar{\varrho})^{4}(x(y, s), s) d s \\
& \leq\left(\varrho_{0}(y)-\bar{\varrho}\right)^{4}+\frac{4 C_{\varepsilon}}{\lambda+2 \mu} \int_{0}^{t}\left(F^{4}+(\vartheta-\bar{\vartheta})^{4}\right)(x(y, s), s) d s .
\end{aligned}
$$

Applying Lemma 3.1 and using the definitions of $F$ and $A(T)$ and the inequality $\|w\|_{L^{4}} \leq C\|w\|^{1 / 4}\left\|\nabla_{x} w\right\|^{3 / 4}$, we have

$$
\begin{aligned}
& \int_{0}^{t}\left(F^{4}+(\vartheta-\bar{\vartheta})^{4}\right)(x(y, s), s) d s \\
& \leq \int_{0}^{t}\left(\|F\|\left\|\nabla_{x} F\right\|^{3}+\|\vartheta-\bar{\vartheta}\|\left\|\nabla_{x} \vartheta\right\|^{3}\right)(x(y, s), s) d s \\
& \leq C \int_{0}^{t}\left\{\left(\left\|\nabla_{x} \vec{u}\right\|+\|\varrho-\bar{\varrho}\|+\|\vartheta-\bar{\vartheta}\|\right)\right. \\
& \left.\left.\quad \times\left(\|\dot{\vec{u}}\|^{3}+\|\vartheta-\bar{\vartheta}\|_{L^{3}}^{3}+\|I-\bar{I}\|_{L^{3}}^{3}\right)\right\} x(y, s), s\right) d s \\
& +C \int_{0}^{t}\left\{\|\vartheta-\bar{\vartheta}\|\left\|\nabla_{x} \vartheta\right\|^{3}\right\}(x(y, s), s) d s \\
& \leq C A(T) \int_{0}^{t}\left(\|\dot{\vec{u}}\|^{2}+\left\|\nabla_{x} \vartheta\right\|^{2}+\|\vartheta-\bar{\vartheta}\|^{2}+\|I-\bar{I}\|_{2}\right) d s \\
& \leq C A^{2}(T), \text { for } t \in[0, T] .
\end{aligned}
$$

Plugging into (3.14), integrating on $\mathbb{R}^{3}$ and using Lemma 2.1, we get (3.10).

2. Suppose first that $T \leq 1$ and integrate (3.12) on trajectories. We find

$$
\begin{aligned}
\|\varrho-\bar{\varrho}\|_{L^{\infty}} \leq & \left\|\varrho_{0}-\bar{\varrho}\right\|_{L^{\infty}} \\
& +\frac{\bar{\varrho}}{\lambda+2 \mu} \int_{0}^{t}\left(\|F\|_{L^{\infty}}+C_{1}\|\varrho-\bar{\varrho}\|_{L^{\infty}}+C_{2}\|\vartheta-\bar{\vartheta}\|_{L^{\infty}}\right) d s,
\end{aligned}
$$

so

$$
\begin{aligned}
\|\varrho-\bar{\varrho}\|_{L^{\infty}} \leq & e_{0}+C \int_{0}^{t}\|\varrho-\bar{\varrho}\|_{L^{\infty}} d s \\
& +C \int_{0}^{t}\left(\|F\|_{L^{\infty}}+\|\vartheta-\bar{\vartheta}\|_{L^{\infty}}\right) d s
\end{aligned}
$$


for $0 \leq t \leq T \leq 1$. The last term is bounded as follows:

$$
\begin{aligned}
& \int_{0}^{t}\left(\|F\|_{L^{\infty}}+\|\vartheta-\bar{\vartheta}\|_{L^{\infty}}\right) d s \\
& \leq C \int_{0}^{t}\left(\|F\|_{L^{4}}+\|\dot{\vec{u}}\|_{L^{4}}+\|\vartheta-\bar{\vartheta}\|_{H^{2}}\right) d s \\
& \leq C \int_{0}^{t}\left\{\left(\left\|\nabla_{x} \vec{u}\right\|^{1 / 4}+\|\varrho-\bar{\varrho}\|^{1 / 4}+\|\vartheta-\bar{\vartheta}\|^{1 / 4}\right)\right. \\
& \left.\quad \times\left(\|\dot{\vec{u}}\|^{3 / 4}+\|\vartheta-\bar{\vartheta}\|^{3 / 4}+\|I-\bar{I}\|^{3 / 4}\right)\right\} d s \\
& \quad+\int_{0}^{t}\|\dot{\vec{u}}\|^{1 / 4}\left\|\nabla_{x} \dot{\vec{u}}\right\|^{3 / 4} d s+C\left(\int_{0}^{t}\left(\|\vartheta-\bar{\vartheta}\|^{2}+\left\|\nabla_{x} \vartheta\right\|^{2}+\|\Delta \vartheta\|^{2}\right) d s\right)^{1 / 2} \\
& \leq C A(T)^{1 / 8}\left(\int_{0}^{t}\left(\|\dot{\vec{u}}\|^{2}\right) d s\right)^{3 / 8} \\
& \quad+C\left(\int_{0}^{t}\left(\|\dot{\vec{u}}\|^{2}\right) d s\right)^{1 / 8}\left(\int_{0}^{t} \phi^{-3 / 4}(s) d s\right)^{1 / 2}\left(\int_{0}^{t}\left(\phi\left\|\nabla_{x} \dot{\vec{u}}\right\|^{2}\right) d s\right)^{3 / 8} \\
& \quad+C A(T)^{1 / 2} \\
& \leq C A(T)^{1 / 2} .
\end{aligned}
$$

Plugging into (3.15) and using Gronwall's inequality we get (3.11) when $T \leq 1$. For $T \geq 1$, multiplying (3.12) by $\varrho-\bar{\varrho}$, we get

$$
\frac{1}{2}(\lambda+2 \mu) \frac{d}{d t}(\varrho-\bar{\varrho})^{2}=-\varrho(\varrho-\bar{\varrho})^{2} F-\varrho(p-\bar{p})(\varrho-\bar{\varrho})^{2} .
$$

We get after (3.1)

$$
\frac{d}{d t}(\varrho-\bar{\varrho})^{2}+\frac{\bar{\varrho} C_{1}}{\lambda+2 \mu}(\varrho-\bar{\varrho})^{2} \leq C\left(\|F\|_{L^{\infty}}^{2}+C_{2}\|\vartheta-\bar{\vartheta}\|_{L^{\infty}}^{2}\right) .
$$

Reasoning as above by integrating on a fixed particle trajectory $x(y, t)$ and using Lemma 2.1, we get

$$
\|\varrho-\bar{\varrho}\|_{L^{\infty}}^{2} \leq\left\|\varrho_{0}-\bar{\varrho}\right\|_{L^{\infty}}^{2}+C \int_{0}^{t} e^{-\beta(t-s)}\left(\|F\|_{L^{4}}^{2}+\|\dot{\vec{u}}\|_{L^{4}}^{2}+\|\vartheta-\bar{\vartheta}\|_{L^{4}}^{2}\right) d s,
$$


with $\beta=\frac{\bar{\varrho} C_{1}}{\lambda+2 \mu}$. Then

$$
\begin{aligned}
\|\varrho-\bar{\varrho}\|_{L^{\infty}}^{2} \leq & \left\|\varrho_{0}-\bar{\varrho}\right\|_{L^{\infty}}^{2}+C \sup _{t \in[1, T]}\left(\|F\|^{1 / 2}\|\dot{\vec{u}}\|^{3 / 2}+\|\vartheta-\bar{\vartheta}\|_{H^{1}}^{2}\right)(t) \\
& +C \int_{0}^{t}\left(\|\dot{\vec{u}}\|^{2}+\left\|\nabla_{x} \dot{\vec{u}}\right\|^{2}\right) d s \leq C\left(e_{0}^{2}+A(T)\right),
\end{aligned}
$$

for $1 \leq t \leq T$, and estimate (3.11) then also follows for $T \geq 1$.

\subsection{Estimates for the radiative quantities}

Making use of the "cut-off" hypothesis (2.2), we first deduce a uniform bound

$$
\begin{aligned}
0 & \leq I(t, x, v, \vec{\omega}) \\
& \leq c(T)\left(1+\sup _{x \in \Omega, \nu \geq 0, \vec{\omega} \in \mathcal{S}^{2}} I_{0}\right) \\
& \leq c(T)\left(1+I_{0}\right) \text { for any } t \in[0, T] .
\end{aligned}
$$

Observe that hypothesis (2.4) together with (3.17) yield

$$
\left\|S_{E}\right\|_{L^{\infty}((0, T) \times \Omega)}+\left\|\vec{S}_{F}\right\|_{L^{\infty}\left((0, T) \times \Omega ; \mathbb{R}^{3}\right)} \leq C,
$$

which, combined with hypothesis (3.1), implies

$$
\left\|\frac{1}{\vartheta} S_{E}\right\|_{L^{\infty}((0, T) \times \Omega)}+\left\|\frac{1}{\vartheta} \vec{S}_{F}\right\|_{L^{\infty}\left((0, T) \times \Omega ; \mathbb{R}^{3}\right)} \leq C .
$$

Finally using a result concerning the velocity averages over the sphere $\mathcal{S}^{2}$ established by Golse et al. [23,24], see also Bournaveas and Perthame [5], and hypothesis (2.4), we have the following result (see [23]):

Lemma 3.3. Let $I \in L^{q}\left([0, T] \times R^{n+1} \times \mathcal{S}^{2}\right), \partial_{t} I+\omega \cdot \nabla_{x} I \in L^{q}\left([0, T] \times R^{n+1} \times\right.$ $\left.\mathcal{S}^{2}\right)$ for a certain $q>1$. In addition, let $I_{0} \equiv I(0, \cdot) \in L^{\infty}\left(R^{n+1} \times \mathcal{S}^{2}\right)$. Then

$$
\tilde{I} \equiv \int_{\mathcal{S}^{2}} I(\cdot, v) \mathrm{d} \vec{\omega}
$$

belongs to the space $W^{s, q}\left([0, T] \times R^{n+1}\right)$ for any $s, 0<s<\inf \{1 / q, 1-1 / q\}$, and

$$
\|\tilde{I}\|_{W^{s, q}} \leq c\left(I_{0}\right)\left(\|I\|_{L^{q}}+\left\|\partial_{t} I+\omega \cdot \nabla I\right\|_{L^{q}}\right) .
$$

Remark 3.4. Note that improved average lemma techniques have been developped in a recent work of Berthelin and Junca [4]. 


\section{Lemma 3.5.}

1. $\sup _{t \geq 0} \int_{\mathbb{R}^{3}} \int_{0}^{\infty} \int_{\mathcal{S}^{2}}|I-\bar{I}|^{4} d \vec{\omega} d \nu d x$

$$
+\int_{0}^{T} \int_{\mathbb{R}^{3}} \int_{0}^{\infty} \int_{\mathcal{S}^{2}}|I-\bar{I}|^{4} d \vec{\omega} d v d x d t \leq C\left(e_{0}^{4}+A^{2}(T)\right),
$$

2. $\sup _{t \geq 0}\left\|E^{R}-\overline{E^{R}}\right\|_{L^{\infty}} \leq C\left(e_{0}+A^{1 / 2}(T)\right)$.

Proof. The proof is very similar to that of Lemma 3.2, so we only give the main lines.

1. From the transport equation (1.6), we have

$$
\begin{aligned}
& \frac{1}{4} \partial_{t}(I-\bar{I})^{4}+\frac{c}{4} \operatorname{div}_{x}\left(\vec{\omega} \cdot \nabla_{x}(I-\bar{I})^{4}\right)+\left(\sigma_{a}+\sigma_{s}\right)(I-\bar{I})^{4} \\
= & \sigma_{a}(B-\bar{B})(I-\bar{I})^{3}+\sigma_{s}(\tilde{I}-\bar{I})(I-\bar{I})^{3} .
\end{aligned}
$$

Integrating with respect to $\vec{\omega}, v$ and $x$ and using Young inequality we find

$$
\begin{aligned}
& \sup _{t \geq 0} \int_{\mathbb{R}^{3}} \int_{0}^{\infty} \int_{\mathcal{S}^{2}}|I-\bar{I}|^{4} d \vec{\omega} d \nu d x \\
& \quad+\int_{0}^{T}\left(\sigma_{a}+\sigma_{s}\right) \int_{\mathbb{R}^{3}} \int_{0}^{\infty} \int_{\mathcal{S}^{2}}|I-\bar{I}|^{4} d \vec{\omega} d \nu d x d t \\
& \leq \varepsilon \int_{0}^{T} \sigma_{a} \int_{\mathbb{R}^{3}} \int_{0}^{\infty} \int_{\mathcal{S}^{2}}|I-\bar{I}|^{4} d \vec{\omega} d \nu d x d t \\
& \quad+C_{\varepsilon} \int_{0}^{T} \int_{\mathbb{R}^{3}}|\vartheta-\bar{\vartheta}|^{4} d x d s .
\end{aligned}
$$

Observing that

$$
\int_{0}^{T}\|\vartheta-\bar{\vartheta}\|_{L^{4}}^{4} d s \leq \int_{0}^{T}\|\vartheta-\bar{\vartheta}\|^{2}\left\|\nabla_{x} \vartheta\right\|^{3} d s \leq C A(T) \int_{0}^{T}\left\|\nabla_{x} \vartheta\right\|^{2} d s,
$$

we get (3.20).

2. Suppose first that $T \leq 1$. From the transport equation (1.6), we have

$$
\partial_{t}(I-\bar{I})+\operatorname{div}_{x}\left(c \vec{\omega} \cdot \nabla_{x} I\right)=\sigma_{a}(B-\bar{B})-\sigma_{a}(I-\bar{I})+\sigma_{s}(\tilde{I}-I) .
$$

Integrating with respect to $\vec{\omega}, v$ and using the characteristics, we have

$$
\left\|\tilde{E}^{R}-\overline{E^{R}}\right\|_{L^{\infty}} \leq\left\|\tilde{E}_{0}^{R}-\overline{E^{R}}\right\|_{L^{\infty}}+C \int_{0}^{t}\|\vartheta-\bar{\vartheta}\|_{L^{\infty}} d s .
$$


Observing that

$$
\begin{aligned}
\int_{0}^{t}\|\vartheta-\bar{\vartheta}\|_{L^{\infty}} d s & \leq \int_{0}^{t}\|\vartheta-\bar{\vartheta}\|_{H^{2}} d s \\
& \leq C\left(\int_{0}^{t}\left(\|\vartheta-\bar{\vartheta}\|^{2}+\left\|\nabla_{x} \vartheta\right\|^{2}+\|\Delta \vartheta\|^{2}\right) d s\right)^{1 / 2} \\
& \leq C A(T)^{1 / 2}
\end{aligned}
$$

we get

$$
\left\|\tilde{E}^{R}-\overline{E^{R}}\right\|_{L^{\infty}} \leq C A(T)^{1 / 2} .
$$

Plugging in (3.23), we find (3.21). For $1 \leq t \leq T \geq 1$, using the method of characteristics, multiplying (3.22) by $e^{\zeta t}(I-\bar{I})$ and integrating with respect to $\vec{\omega}, v$ and $t$, we get

$$
\left\|E^{R}(t)-\overline{E^{R}}\right\|_{L^{\infty}}^{2} \leq\left\|E^{R}(1)-\overline{E^{R}}\right\|_{L^{\infty}}^{2}+C \int_{1}^{t} e^{-\zeta(t-s)}\|\vartheta-\bar{\vartheta}\|_{L^{\infty}} d s,
$$

with $\zeta=\frac{1}{2}\left(\underline{\sigma_{a}}+\underline{\sigma_{s}}\right)$. Then

$$
\left\|E^{R}-\overline{E^{R}}\right\|_{L^{\infty}}^{2} \leq\left\|E^{R}(1)-\overline{E^{R}}\right\|_{L^{\infty}}^{2}+C \sup _{s \in[1, T]}\left(\|\vartheta-\bar{\vartheta}\|_{H^{1}}^{2}(t)+\|\Delta \vartheta\|^{2}\right)(s)
$$

so we conclude that

$$
\left\|E^{R}-\overline{E^{R}}\right\|_{L^{\infty}}^{2} \leq C\left(e_{0}^{2}+A(T)\right)
$$

for $1 \leq t \leq T$, and estimate (3.21) then also follows for $T \geq 1$.

\section{3. $L^{p}$-estimates for vorticity, velocity and temperature}

In the spirit of [29], we have vorticity-velocity estimates. We will adopt the slightly incorrect notation $\omega^{p}$ for $\left(\omega_{i k}\right)^{p}$

Lemma 3.6. The following bounds hold:

1. $\sup _{t \in[0, T]}\|\omega\|_{L^{4}}^{4}+\int_{0}^{T}\left(\|\omega\|_{L^{4}}^{4}+\|\omega\|_{L^{6}}^{6}\right) d t \leq C\left(e_{0}^{2}+A^{4 / 3}+A^{3}\right)$,

2 .

$$
\int_{0}^{T}\left\|\nabla_{x} \vec{u}\right\|_{L^{4}}^{4} d t \leq C\left(e_{0}^{2}+A^{4 / 3}+A^{3}\right)
$$




\section{Proof.}

1. Applying $\partial_{k}$ to equation (1.4), we get

$$
\begin{aligned}
\rho \frac{d}{d t} \partial_{k} u_{i}= & \mu \Delta \partial_{k} u_{i}+(\lambda+\mu) \partial_{k} \partial_{i} \operatorname{div}_{x} \vec{u} \\
& -\partial_{k} \varrho \dot{u}_{i}-\varrho \partial_{k}\left(\left(\vec{u} \cdot \nabla_{x}\right) u_{i}\right)-\partial_{k} \partial_{i} p-\partial_{k} S_{F i} .
\end{aligned}
$$

Subtracting the same relation with $k \leftrightarrow i$ we have

$$
\begin{aligned}
\rho \frac{d}{d t} \omega_{i k}= & \mu \Delta \omega_{i k}-\partial_{k} \varrho \dot{u_{i}}+\partial_{i} \varrho \dot{u_{k}} \\
& +\varrho\left[\partial_{i}\left(\vec{u} \cdot \nabla_{x}\right) u_{k}-\partial_{k}\left(\vec{u} \cdot \nabla_{x}\right) u_{i}\right]-\partial_{k} S_{F i}+\partial_{i} S_{F k} .
\end{aligned}
$$

Multiplying by $(\omega: \omega) \omega_{i k}$ and integrating by parts on $[0, t] \times \mathbb{R}^{3}$, we get

$$
\begin{aligned}
& \|\omega\|_{L^{4}}^{4}+\int_{0}^{t} \int \omega^{2}\left|\nabla_{x} \omega\right|^{2} d x d s \\
& \leq C\left(e_{0}^{2}+A^{2}\right) \\
& \quad+C \int_{0}^{t} \int\left(\left|\nabla_{x} \vec{u}\right|^{2}|\omega|^{3}+|\dot{\vec{u}}||\omega|^{2}\left|\nabla_{x} \omega\right|+|\omega|^{3}\left|\nabla_{x} \dot{\vec{u}}\right|\right) d x d s \\
& \quad+C\left|\int_{0}^{t} \int \omega^{3}\left(\partial_{k} S_{F i}-\partial_{i} S_{F k}\right) d x d s\right| .
\end{aligned}
$$

After the definition of $F$, using the Gagliardo-Nirenberg inequality, we find

$$
\begin{aligned}
\int_{0}^{t} \int \omega^{4} d x d s & \leq \int_{0}^{t} \int\left(|\omega|^{10 / 3}+|\omega|^{6}\right) d x d s \\
& \leq C \int_{0}^{t}\left(\|\omega\|^{4 / 3}\left\|\nabla_{x} \omega\right\|^{2}+\|\omega\|^{6}\right) d s \\
& \leq C A^{5 / 3}+C \int_{0}^{t}\|\omega\|^{6} d s .
\end{aligned}
$$

So, adding $\int_{0}^{t} \int\left(|\omega|^{4}+|\omega|^{6}\right) d x d s$ to (3.27), we get

$$
\begin{aligned}
& \|\omega\|_{L^{4}}^{4}+\int_{0}^{t} \int \omega^{2}\left(|\omega|^{2}+|\omega|^{3}+\left|\nabla_{x} \omega\right|^{2}\right) d x d s \\
& \leq C\left(e_{0}^{2}+A^{2}+A^{5 / 3}\right)+\varepsilon \int_{0}^{t}\left\|\nabla_{x} \vec{u}\right\|_{L^{4}}^{4} d s+\frac{C}{\varepsilon} \int_{0}^{t}\|\omega\|^{6} d s \\
& \quad+C \int_{0}^{t} \int\left(|\dot{\vec{u}}|^{2}|\omega|^{2}+|\omega|^{3}\left|\nabla_{x} \dot{\vec{u}}\right|\right) d x d s \\
& \quad+\left|\int_{0}^{t} \int(\omega: \omega) \omega_{i k}\left(\partial_{k} S_{F i}-\partial_{i} S_{F k}\right) d x d s\right|=: \Omega .
\end{aligned}
$$


Integrating by parts in $\left.J:=\int_{0}^{t} \int \omega^{2} \omega_{i k}\left(\partial_{k}\left(S_{F}-\overline{S_{F}}\right)_{i}\right)-\partial_{i}\left(S_{F}-\overline{S_{F}}\right)_{k}\right) \mid d x d s$, we see that

$$
J=-2 \int_{0}^{t} \int \partial_{k}\left(\omega_{\alpha \beta} \omega_{\alpha \beta} \omega_{i k}\right)\left(S_{F}-\overline{S_{F}}\right)_{i} d x d s
$$

Then

$$
|J| \leq C\left[e_{0}^{2}+\sum_{j} \int_{0}^{t} \int\left|\partial_{k}\left(\omega_{\alpha \beta} \omega_{\alpha \beta} \omega_{i k}\right)\right|^{2}\left(S_{F}-\overline{S_{F}}\right)_{i} d x d s\right]
$$

and using (3.21)

$$
\begin{gathered}
|J| \leq C\left[e_{0}^{2}+\sup _{[0, T]}\left\{\int|\omega|^{4} d x \cdot \int_{0}^{t} \int\left|\nabla_{x} \omega\right|^{2} d x d s+\left\|E^{R}-\overline{E^{R}}\right\|_{L^{\infty}}\right\}\right] \\
\leq C\left(e_{0}^{2}+A^{2}\right) .
\end{gathered}
$$

Plugging into (3.28), we get

$$
\begin{aligned}
|\Omega| \leq & C\left(e_{0}^{2}+A^{2}+A^{5 / 3}\right)+\varepsilon \int_{0}^{t}\|\omega\|_{L^{4}}^{4} d s+\frac{C}{\varepsilon} \int_{0}^{t}\|\omega\|^{6} d s \\
& +C\left(\int_{0}^{t} \int\|\dot{\vec{u}}\|_{L^{3}}^{3} d s\right)^{2 / 3}\left(\int_{0}^{t}\|\omega\|^{6} d s\right)^{1 / 3}+C A^{1 / 2}\left(\int_{0}^{t}\|\omega\|^{6} d s\right)^{1 / 2} .
\end{aligned}
$$

Applying now Gagliardo Nirenberg's inequality, we get

$$
\int_{0}^{t} \int\|\omega\|_{L^{6}}^{6} d x \leq C A^{3 / 4} \int_{0}^{t} \int\left\|\omega \mid \nabla_{x} \omega\right\|^{2} d x,
$$

and

$$
\int_{0}^{t} \int\|\dot{\vec{u}}\|_{L^{3}}^{3} d x \leq C A^{3 / 2}
$$

then plugging (3.29) and (3.30) into (3.28), we obtain

$$
\|\omega\|_{L^{4}}^{4}+\int_{0}^{t}\left(\|\omega\|_{L^{4}}^{4}+\|\omega\|_{L^{6}}^{6}\right) d s \leq \frac{C}{\varepsilon^{4}}\left(e_{0}^{2}+A^{3}+A^{4 / 3}\right)+\varepsilon \int_{0}^{t}\|\omega\|_{L^{4}}^{4} d s
$$

and taking $\varepsilon$ small enough, we get (3.24).

2. After Lemma 3.1

$$
\left\|\nabla_{x} \vec{u}\right\|_{L^{4}} \leq C\left(\|F\|_{L^{4}}+\|\omega\|_{L^{4}}+\|\varrho-\bar{\varrho}\|_{L^{4}}+\|\varrho-\bar{\varrho}\|_{L^{4}}\right),
$$

and

$$
\int_{0}^{t}\left\|\nabla_{x} \vec{u}\right\|_{L^{4}} d s \leq C\left(e_{0}^{2}+A^{2}\right)+C \int_{0}^{t}\|\omega\|_{L^{4}}^{4} d s .
$$

Using (3.24) gives (3.25). 
Lemma 3.7. The following bound holds:

$$
\begin{aligned}
& \sup _{t \in[0, T]}\left(\|\varrho-\bar{\varrho}\|^{2}+\|\vec{u}\|^{2}+\|\vartheta-\bar{\vartheta}\|^{2}+\left\|E^{R}-\overline{E^{R}}\right\|^{2}\right) \\
& \quad+\int_{0}^{T}\left(\left\|\nabla_{x} \vec{u}\right\|^{2}+\left\|\nabla_{x} \vartheta\right\|^{2}\right) d t \\
& \leq C\left(e_{0}^{2}+A^{4 / 3}+A^{3}\right) .
\end{aligned}
$$

\section{Proof.}

1. Let us consider first the case $T \leq 1$. Multiplying (1.4) and (1.5) respectively by $\vec{u}$ and $\vartheta-\bar{\vartheta}$ and integrating by parts on $[0, T] \times \mathbb{R}^{3}$, we get

$$
\begin{aligned}
& \|\vec{u}\|^{2}+\|\vartheta-\bar{\vartheta}\|^{2}+\int_{0}^{t}\left(\left\|\nabla_{x} \vec{u}\right\|^{2}+\left\|\nabla_{x} \vartheta\right\|^{2}\right) d s \\
& \leq C e_{0}^{2}+\int_{0}^{t}\left(\|\varrho-\bar{\varrho}\|^{2}+\|\vartheta-\bar{\vartheta}\|^{2}\right) d s \\
& \quad+C \int_{0}^{t} \int\left(\left|\nabla_{x} \vec{u}\right|^{4}+\|\vartheta-\bar{\vartheta}\|^{4}\right) d x d s \\
& +\left|\int_{0}^{t} \int(\vartheta-\bar{\vartheta}) S_{E} d x d s\right|+\left|\int_{0}^{t} \int \vec{u} \cdot \vec{S}_{F} d x d s\right|,
\end{aligned}
$$

where the last two terms are respectively bounded by $C\left(\int_{0}^{t}\|\vartheta-\bar{\vartheta}\|^{2} d s+e_{0}^{2}\right)$ and $C\left(\int_{0}^{t}\|\vec{u}\|^{2} d s+e_{0}^{2}\right)$. Multiplying the equation $\dot{\varrho}=-\varrho \operatorname{div}_{x} \vec{u}$ by $\varrho(\varrho-\bar{\varrho})$ and integrating on $[0, T] \times \mathbb{R}^{3}$, we get

$$
\|\varrho-\bar{\varrho}\|^{2} \leq C \int_{0}^{t}\|\varrho-\bar{\varrho}\|^{2} d s+\frac{1}{2} \int_{0}^{t}\left\|\nabla_{x} \vec{u}\right\|^{2} d s
$$

Adding to (3.32) and applying Gronwall's inequality, we find

$$
\begin{aligned}
& \|\varrho-\bar{\varrho}\|^{2}+\|\vec{u}\|^{2}+\|\vartheta-\bar{\vartheta}\|^{2}+\int_{0}^{t}\left(\left\|\nabla_{x} \vec{u}\right\|^{2}+\left\|\nabla_{x} \vartheta\right\|^{2}\right) d s \\
& \leq C\left(e_{0}^{2}+A^{4 / 3}+A^{3}\right),
\end{aligned}
$$

for any $0 \leq t \leq T \leq 1$.

2. When $T \geq 1$, we consider [21] the Helmholtz function

$$
H_{\vartheta}(\varrho, \vartheta)=\varrho[e(\varrho, \vartheta)-\bar{\vartheta} s(\varrho, \vartheta)]
$$


and from (1.7) we have the energy identity

$$
\begin{aligned}
& \frac{d}{d t} \int\left[\frac{1}{2} \vec{u}^{2}+E^{R}+H_{\bar{\vartheta}}(\varrho, \vartheta)-(\varrho-\bar{\varrho}) \partial_{\varrho} H_{\bar{\vartheta}}(\bar{\varrho}, \bar{\vartheta})-H_{\bar{\vartheta}}(\bar{\varrho}, \bar{\vartheta})-\bar{\vartheta} \varrho s\right] d x \\
& =-\int \vec{u} \cdot \vec{S}_{F} d x .
\end{aligned}
$$

From the equation of entropy we get

$$
\begin{aligned}
\frac{d}{d t} \int \bar{\vartheta} \rho s d x= & \int\left\{\frac{\bar{\vartheta}}{\vartheta}\left[\mu\left(\operatorname{div}_{x} \vec{u}\right)^{2}+2 \mu \mathbb{D}: \mathbb{D}\right]+\kappa \bar{\vartheta} \frac{\left|\nabla_{x} \vartheta\right|^{2}}{\vartheta^{2}}\right\} d x \\
& +\int \frac{\bar{\vartheta}}{\vartheta}\left(\vec{u} \cdot \vec{S}_{F}-S_{E}\right) d x .
\end{aligned}
$$

Recalling the definition of the radiative entropy production rate

$$
\begin{aligned}
\bar{\vartheta} \varsigma^{R}= & \bar{\vartheta} \frac{S_{E}}{\vartheta}-\bar{\vartheta} \frac{\vec{u} \cdot \vec{S}_{F}}{\vartheta} \\
& -\frac{k \bar{\vartheta}}{h} \int_{0}^{\infty} \int_{\mathcal{S}^{2}} \frac{1}{v}\left[\log \frac{n(I)}{n(I)+1}-\log \frac{n(B)}{n(B)+1}\right] \sigma_{a}(B-I) d \vec{\omega} d v \\
& -\frac{k \bar{\vartheta}}{h} \int_{0}^{\infty} \int_{\mathcal{S}^{2}} \frac{1}{v}\left[\log \frac{n(I)}{n(I)+1}-\log \frac{n(\tilde{I})}{n(\tilde{I})+1}\right] \sigma_{s}(\tilde{I}-I) d \vec{\omega} d v
\end{aligned}
$$

plugging into (3.33) and integrating on $[1, t] \times \mathbb{R}^{3}$, we find

$$
\begin{aligned}
& \int\left[\frac{1}{2} \vec{u}^{2}+H_{\bar{\vartheta}}(\varrho, \vartheta)-(\varrho-\bar{\varrho}) \partial_{\varrho}-H_{\bar{\vartheta}}(\bar{\varrho}, \bar{\vartheta})+E^{R}-\bar{\vartheta}^{R}-\overline{E^{R}}\right] d x \\
& +\bar{\vartheta} \int_{1}^{t} \int\left\{\frac{\bar{\vartheta}}{\vartheta} \mu\left(\operatorname{div}_{x} \vec{u}\right)^{2}+2 \mu \mathbb{D}: \mathbb{D}+\kappa \bar{\vartheta} \frac{\left|\nabla_{x} \vartheta\right|^{2}}{\vartheta^{2}}\right\} d x d s \\
& +\frac{k \bar{\vartheta}}{h} \int_{1}^{t} \iint_{0}^{\infty} \int_{\mathcal{S}^{2}} \frac{1}{v}\left[\log \frac{n(I)}{n(I)+1}-\log \frac{n(B)}{n(B)+1}\right] \sigma_{a}(B-I) d \vec{\omega} d v d x d s \\
& +\frac{k \bar{\vartheta}}{h} \int_{1}^{t} \iint_{0}^{\infty} \int_{\mathcal{S}^{2}} \frac{1}{v}\left[\log \frac{n(I)}{n(I)+1}-\log \frac{n(\tilde{I})}{n(\tilde{I})+1}\right] \sigma_{s}(\tilde{I}-I) d \vec{\omega} d v d x d s \\
& =\int\left[\frac{1}{2} \vec{u}^{2}+H_{\bar{\vartheta}}(\varrho, \vartheta)-(\varrho-\bar{\varrho}) \partial_{\varrho}-H_{\bar{\vartheta}}(\bar{\varrho}, \bar{\vartheta})+E^{R}-\bar{\vartheta} s^{R}\right]_{t=1} d x \\
& \quad-\int \vec{u} \cdot \vec{S}_{F} d x .
\end{aligned}
$$

Using the convexity of the functions $H_{\bar{\vartheta}}(\varrho, \vartheta)-(\varrho-\bar{\varrho}) \partial_{\varrho}-H_{\bar{\vartheta}}(\bar{\varrho}, \bar{\vartheta})$ and 
$E^{R}-\bar{\vartheta}^{R}-\overline{E^{R}}$ and hypothesis (3.1), we conclude that

$$
\begin{aligned}
& \sup _{t \in[0, T]}\left(\|\varrho-\bar{\varrho}\|^{2}+\|\vec{u}\|^{2}+\|\vartheta-\bar{\vartheta}\|^{2}+\left\|E^{R}-\overline{E^{R}}\right\|^{2}\right) \\
& +\int_{0}^{T}\left(\left\|\nabla_{x} \vec{u}\right\|^{2}+\left\|\nabla_{x} \vartheta\right\|^{2}\right) d t \leq C\left(e_{0}^{2}+A^{4 / 3}+A^{3}\right)
\end{aligned}
$$

for $T \geq 1$.

\subsection{Estimates for derivatives}

Lemma 3.8. The following bounds hold:

1. $\sup _{t \in[0, T]}\left(\left\|\nabla_{x} \vec{u}\right\|^{2}+\left\|\nabla_{x} \vartheta\right\|^{2}\right)+\int_{0}^{T}\left(\|\dot{\vec{u}}\|^{2}+\|\dot{\vartheta}\|^{2}\right) d t$

$\leq C\left(e_{0}^{2}+A^{4 / 3}+A^{3}\right)$

2. $\quad \int_{0}^{T}\|\Delta \vartheta\|^{2} d t \leq C\left(e_{0}^{2}+A^{4 / 3}+A^{3}\right)$,

3. $\quad \int_{0}^{T}\left\|\nabla_{x} \omega\right\|^{2} d t \leq C\left(e_{0}^{2}+A^{4 / 3}+A^{3}\right)$.

Proof.

1. Using the formula

$$
\int_{0}^{t} \int f \dot{g} d x d s=\left.\int f g d x\right|_{0} ^{t}-\int_{0}^{t} \int \dot{f} g d x d s-\int_{0}^{t} \int f g \operatorname{div}_{x} \vec{u} d x d s,
$$

multiplying (1.4) by $\dot{\vec{u}}$ and (1.5) by $\dot{\vartheta}$, adding and integrating by parts, we get

$$
\begin{aligned}
& \int \varrho\left(\dot{\vec{u}}^{2}+e_{\vartheta} \dot{\vartheta}^{2}\right) d x \\
& \leq-\frac{d}{d t} \int\left\{\mu\left|\nabla_{x} \vec{u}\right|^{2}+(\lambda+\mu)\left(\operatorname{div}_{x} \vec{u}\right)^{2}+\kappa\left|\nabla_{x} \vartheta\right|^{2}\right\} d x \\
& \quad-\int\left(\nabla_{x} p \cdot \dot{\vec{u}}+\dot{\vartheta} S_{E}+\dot{\vec{u}} \cdot \vec{S}_{F}\right) d x \\
& \quad+C \int\left\{\vartheta^{2}\left|\nabla_{x} \vec{u}\right|^{2}+\left|\nabla_{x} \vec{u}\right|^{4}+\left|\nabla_{x} \vec{u}\right|\left|\nabla_{x} \vartheta\right|^{2}+\left|\nabla_{x} \vec{u}\right|^{3}\right\} d x .
\end{aligned}
$$


Integrating in $t$ and using Young's inequality we have

$$
\begin{aligned}
& \frac{\bar{\varrho}}{2} \int_{0}^{t}\left(\|\dot{\vec{u}}\|^{2}+\underline{e}_{\vartheta} \dot{\vartheta}^{2}\right) d x \leq C e_{0}^{2}-\frac{1}{2}\left(\mu\left\|\nabla_{x} \vec{u}\right\|^{2}+\kappa\left\|\nabla_{x} \vartheta\right\|^{2}\right) \\
& \quad+\int_{0}^{t} \int\left\{(p-\bar{p}) \operatorname{div}_{x} \dot{\vec{u}}-S_{E} \dot{\vartheta}\right\} d x \\
& \quad+C \int_{0}^{t} \int\left(|\vartheta-\bar{\vartheta}|^{4}+\left|\nabla_{x} \vec{u}\right|^{2}+\left|\nabla_{x} \vec{u}\right|^{4}+\left|\nabla_{x} \vartheta\right|^{8 / 3}\right) d x d s .
\end{aligned}
$$

Now observe that

$$
\begin{aligned}
& \int_{0}^{t} \int(p-\bar{p}) \operatorname{div}_{x} \dot{\vec{u}} d x \\
& \leq \int_{0}^{t} \int(p-\bar{p}) \frac{d}{d t} \operatorname{div}_{x} \vec{u} d x d s+\int_{0}^{t} \int(1+|\vartheta-\bar{\vartheta}|)\left|\nabla_{x} \vec{u}\right|^{2} d x d s \\
& \leq\left.\int(p-\bar{p}) \frac{d}{d t} \operatorname{div}_{x} \vec{u} d x\right|_{0} ^{t}-\int_{0}^{t} \int \dot{p} \operatorname{div}_{x} \vec{u} d x d s \\
& -\int_{0}^{t} \int p\left(\operatorname{div}_{x} \vec{u}\right)^{2} d x d s \\
& \leq\left.\int(p-\bar{p}) \frac{d}{d t} \operatorname{div}_{x} \vec{u} d x\right|_{0} ^{t}+\int_{0}^{t} \int p_{\varrho} \varrho\left(\operatorname{div}_{x} \vec{u}\right)^{2} d x d s \\
& -\int_{0}^{t} \int p_{\vartheta} \dot{\vartheta} \operatorname{div}_{x} \vec{u} d x d s-\int_{0}^{t} \int p\left(\operatorname{div}_{x} \vec{u}\right)^{2} d x d s \\
& \leq C\left(e_{0}^{2}+\|\varrho-\bar{\varrho}\|^{2}+\|\vartheta-\bar{\vartheta}\|^{2}\right)+\frac{1}{4} \mu\left\|\nabla_{x} \vec{u}\right\|^{2} \\
& +\frac{1}{4} \bar{\varrho} \underline{e_{\vartheta}} \int_{0}^{t}\|\dot{\vartheta}\|^{2} d s \\
& +C \int_{0}^{t} \int\left(|\vartheta-\bar{\vartheta}|^{4}+\left|\nabla_{x} \vec{u}\right|^{2}+\left|\nabla_{x} \vec{u}\right|^{4}\right) d x d s .
\end{aligned}
$$

Let us estimate the right-hand side. We first find

$$
\begin{aligned}
\left|\int_{0}^{t} \int(p-\bar{p}) \operatorname{div}_{x} \dot{\vec{u}} d x\right| \leq & C\left(e_{0}^{2}+A^{4 / 3}+A^{3}\right)+\frac{1}{4} \mu\left\|\nabla_{x} \vec{u}\right\|^{2} \\
& +\frac{1}{4} \bar{\varrho} \underline{e_{\vartheta}} \int_{0}^{t}\|\dot{\vartheta}\|^{2} d s .
\end{aligned}
$$


We also have

$$
\begin{aligned}
& \int_{0}^{t} \int\left|\nabla_{x} \vartheta\right|^{8 / 3} d x d s \leq C\left(e_{0}^{2}+A^{4 / 3}+A^{3}\right) \\
&+C \int_{0}^{t}\left\|\nabla_{x} \vartheta\right\|^{4 / 3}\|\Delta \vartheta\|^{2} d s \\
& \leq C\left(e_{0}^{2}+A^{4 / 3}+A^{3}+A^{5 / 3}\right) \\
& \leq C\left(e_{0}^{2}+A^{4 / 3}+A^{3}\right), \\
&\left|\int_{0}^{t} \dot{\vartheta} S_{E} d x d s\right| \leq C e_{0}^{2}+\frac{1}{4} \bar{\varrho} \underline{e_{\vartheta}} \int_{0}^{t}\|\dot{\vartheta}\|^{2} d s
\end{aligned}
$$

and

$$
\left|\int_{0}^{t} \dot{\vec{u}} \vec{S}_{F} d x d s\right| \leq C e_{0}^{2}+\frac{1}{2} \bar{\varrho} \int_{0}^{t}\|\dot{\vec{u}}\|^{2} d s .
$$

Plugging (3.38), (3.39), (3.40) and (3.41) into (3.37), we get (3.34).

2. Multiplying (3.26) by $\omega_{i j}$, integrating by parts and using (3.34) gives estimate (3.35).

3. Finally (3.36) follows from (1.5) and (3.34).

Lemma 3.9. The following bounds hold:

1. $\sup _{t \in[0, T]}\|\dot{\vec{u}}\|^{2}+\int_{0}^{T}\left\|\nabla_{x} \dot{\vec{u}}\right\|^{2} d t \leq C\left(e_{0}^{2}+A^{4 / 3}+A^{3}\right)$,

2. $\sup _{t \in[0, T]} \phi(t)\|\dot{\vartheta}\|^{2}+\int_{0}^{T} \phi(s)\left\|\nabla_{x} \dot{\vartheta}\right\|^{2} d t \leq C\left(e_{0}^{2}+A^{4 / 3}+A^{3}\right)$,

3. $\sup _{t \in[0, T]} \phi^{2}(t)\|\Delta \vartheta\|^{2} \leq C\left(e_{0}^{2}+A^{4 / 3}+A^{3}\right)$.

Proof.

1. Applying $\frac{d}{d t}$ to the momentum equation (1.4), we get

$$
\begin{gathered}
\varrho \ddot{u}_{j}=\mu\left\{\Delta \partial_{t} u_{j}+\operatorname{div}_{x}\left(\vec{u} \Delta u_{j}\right\}+(\lambda+\mu)\left\{\partial_{j}\left(\operatorname{div}_{x} \partial_{t} \vec{u}\right)+\operatorname{div}_{x}\left(\vec{u} \partial_{j} \operatorname{div}_{x} \vec{u}\right)\right\}\right. \\
-\partial_{j} \partial_{t} p+\operatorname{div}_{x}\left(\vec{u} \partial_{j} p\right)-\dot{S}_{F j} .
\end{gathered}
$$


Multiplying by $\dot{\vec{u}}$ and integrating by parts, we get

$$
\begin{aligned}
& \frac{\bar{\varrho}}{2}\left\|\dot{u}_{j}\right\|^{2}+\int_{0}^{t} \int\left(\mu\left|\nabla_{x} \dot{u}_{j}\right|^{2}+(\lambda+\mu)\left(\operatorname{div}_{x} \dot{\vec{u}}\right)^{2}\right) d x d s \\
& =\int_{0}^{t} \int(\lambda+\mu)\left\{\partial_{k} u_{j} \partial_{j} u_{k} \operatorname{div}_{x} \dot{\vec{u}}-\left(\operatorname{div}_{x} \vec{u}\right)^{2}\left(\operatorname{div}_{x} \vec{u}\right)^{2}+\partial_{k} \dot{u}_{j} \partial_{j} u_{k} \operatorname{div}_{x} \vec{u}\right\} d x d s \\
& \quad-\int_{0}^{t} \int\left\{\dot{p} \operatorname{div}_{x} \dot{\vec{u}}+p\left(\operatorname{div}_{x} \vec{u} \operatorname{div}_{x} \dot{\vec{u}}-\partial_{k} \dot{u}_{j} \partial_{j} u_{k}-\dot{u}_{j} \dot{S}_{F} j\right\} d x d s .\right.
\end{aligned}
$$

So we get

$$
\begin{aligned}
& \left\|\dot{u}_{j}\right\|^{2}+\int_{0}^{t} \int\left\|\nabla_{x} \dot{u}_{j}\right\|^{2} d s \\
& \leq C e_{0}^{2}+C \int_{0}^{t} \int\left\{\left|\nabla_{x} \vec{u}\right|^{4}+|\dot{\vec{u}}|^{2}+|\dot{\vartheta}|^{2}+\vartheta^{2}\left|\nabla_{x} \vec{u}\right|^{2}+\left|\nabla_{x} \vec{u}\right|^{2}+\left|\dot{E}^{R}\right|^{2}\right\} d x d s .
\end{aligned}
$$

Using (3.1) and Lemma 3.8, we get (3.42).

2. In the same stroke, applying $\frac{d}{d t}$ to the energy equation (1.5), we get

$$
\begin{aligned}
\varrho e_{\vartheta} \ddot{\vartheta}= & \varrho e_{\vartheta} \dot{\vartheta} \operatorname{div}_{x} \vec{u}-\varrho e_{\varrho \vartheta} \dot{\varrho} \dot{\vartheta}-\varrho e_{\vartheta \vartheta} \dot{\vartheta}^{2}-\left[\vartheta p_{\varrho \vartheta} \dot{\varrho}+\left(p_{\vartheta}+\vartheta p_{\vartheta \vartheta} \dot{\vartheta}\right)\right] \operatorname{div}_{x} \vec{u} \\
& -\vartheta p_{\vartheta} \operatorname{div}_{x} \vec{u}+2 \mu \frac{d}{d t}(\mathbb{D}: \mathbb{D})+\lambda \frac{d}{d t}\left(\operatorname{div}_{x} \vec{u}\right)^{2}+\kappa \frac{d}{d t}(\Delta \vartheta)-\dot{S}_{E},
\end{aligned}
$$

or

$$
\begin{aligned}
\varrho e_{\vartheta} \ddot{\vartheta}= & \varrho e_{\vartheta} \dot{\vartheta} \operatorname{div}_{x} \vec{u}+\varrho^{2} e_{\varrho \vartheta} \dot{\vartheta} \operatorname{div}_{x} \vec{u}-\varrho e_{\vartheta \vartheta} \dot{\vartheta}^{2} \\
& +\varrho \vartheta p_{\varrho \vartheta}\left(\operatorname{div}_{x} \vec{u}\right)^{2}-\left(p_{\vartheta}+\vartheta p_{\vartheta \vartheta}\right) \dot{\vartheta} \operatorname{div}_{x} \vec{u}-\vartheta p_{\vartheta} \operatorname{div}_{x} \vec{u} \\
& +2 \mu D_{i j}\left[\partial_{i} \dot{u}_{j}+\partial_{j} \dot{u}_{i}-\partial_{i} u_{k} \partial_{k} u_{j}-\partial_{j} u_{k} \partial_{k} u_{i}\right] \\
& +2 \lambda \operatorname{div}_{x} \vec{u}\left[\operatorname{div}_{x} \dot{\vec{u}}-\partial_{k} u_{j} \partial_{j} u_{k}\right]+\kappa\left(\Delta \dot{\vartheta}-\Delta \vartheta \operatorname{div}_{x} \vec{u}\right)-\dot{S}_{E} .
\end{aligned}
$$

Observing that

$\varrho e_{\vartheta} \ddot{\vartheta} \phi \dot{\vartheta}=\frac{1}{2} \varrho \frac{d}{d t}\left\{\phi e_{\vartheta} \dot{\vartheta}^{2}\right\}+\frac{1}{2} \varrho \phi^{\prime} e_{\vartheta} \dot{\vartheta}^{2}-\frac{1}{2} \varrho^{2} \phi e_{\varrho \vartheta} \dot{\vartheta}^{2} \operatorname{div}_{x} \vec{u}+\frac{1}{2} \varrho \phi^{\prime} e_{\vartheta \vartheta} \dot{\vartheta}^{3}$,

and that

$$
\dot{S}_{E}=\int_{0}^{\infty} \int_{\mathcal{S}^{2}} c\left\{\left(\partial_{\vartheta} \sigma_{a} \operatorname{div}_{x} \vec{u}-\varrho \partial_{\vartheta} \sigma_{a} \dot{\vartheta}\right)(B-I)+\sigma_{a}\left(\partial_{\vartheta} B \dot{\vartheta}-\dot{I}\right)\right\} d \vec{\omega} d \nu,
$$


multiplying by $\phi(t) \dot{\vartheta}$, integrating by parts on $[0, t] \times \mathbb{R}^{3}$, using (3.1) together with Lemma 3.8 and (3.42) we obtain

$$
\begin{aligned}
& \frac{1}{2} \int \varrho \phi e_{\vartheta} \dot{\vartheta}^{2} d x+\int_{0}^{t} \int \kappa \phi\left|\nabla_{x} \dot{\vartheta}\right|^{2} d x d s \\
& \leq C \int_{0}^{t} \int\left[|\dot{\vartheta}|^{2}+|\dot{I}|^{2}\right] d x d s \\
& \quad+C \int_{0}^{t} \int \phi\left\{| \dot { \vartheta } | \left(|\vartheta|\left|\nabla_{x} \vec{u}\right|^{2}+\left|\nabla_{x} \vec{u}\right|^{3}+|\dot{\vartheta}|^{2}+\left|\nabla_{x} \vec{u} \| \dot{\vartheta}\right|\right.\right. \\
& \left.\left.\quad+|\vartheta|\left|\nabla_{x} \dot{\vec{u}}\right|+\left|\nabla_{x} \vec{u}\right|\left|\nabla_{x} \dot{\vec{u}}\right|\right)+\left|\nabla_{x} \vec{u}\right|^{2}\left|\nabla_{x} \vartheta\right|^{2}\right\} d x d s \\
& \leq C \int_{0}^{t}\left[\|\dot{\vartheta}\|^{2}+\|\dot{I}\|^{2}+\left\|\nabla_{x} \vec{u}\right\|_{L^{4}}^{4}+\phi\left\|\nabla_{x} \dot{\vec{u}}\right\|^{2}\right] d s \\
& +C \int_{0}^{t} \int \phi\left[\left(\left|\nabla_{x} \vec{u}\right|^{2}+|\vartheta-\bar{\vartheta}|^{2}+\left|E^{R}-\overline{E^{R}}\right|^{2}\right)|\dot{\vartheta}|^{2}+\left|\nabla_{x} \vartheta\right|^{2}\right] \mathrm{d} x d s .
\end{aligned}
$$

Now observing that

$$
\int_{0}^{t} \int|\omega|^{5} d x d s \leq \int_{0}^{t}\left(\|\omega\|_{L^{4}}^{4}+\|\omega\|_{L^{6}}^{6}\right) d x d s \leq C\left(e_{0}^{2}+A^{4 / 3}+A^{3}\right),
$$

and using Lemma 3.1, we get

$$
\begin{aligned}
& \int_{0}^{t} \int\left(\left|\nabla_{x} \vec{u}\right|^{5}+|\vartheta-\bar{\vartheta}|^{5}\right) d x d s \\
& \leq \int_{0}^{t}\left(\left\|\nabla_{x} F\right\|_{L^{5}}^{5}+|\vartheta-\bar{\vartheta}|_{L^{5}}^{5}+\left|E^{R}-\overline{E^{R}}\right|_{L^{5}}^{5}+\|\omega\|_{L^{5}}^{5}+|\varrho-\bar{\varrho}|_{L^{4}}^{4}\right) d x d s \\
& \leq C\left(e_{0}^{2}+A^{4 / 3}+A^{3}\right)+C A^{1 / 4} \int_{0}^{t}\left(\|\dot{\vec{u}}\|^{2}+\left|\nabla_{x} \vartheta\right|^{2}\right)^{9 / 4} d x d s \\
& \leq C\left(e_{0}^{2}+A^{4 / 3}+A^{3}\right),
\end{aligned}
$$

so we can bound the second term in (3.45) as follows:

$$
\begin{aligned}
& \int_{0}^{t} \int \phi\left[\left(\left|\nabla_{x} \vec{u}\right|^{2}+|\vartheta-\bar{\vartheta}|^{2}+\left|E^{R}-\overline{E^{R}}\right|^{2}\right)|\dot{\vartheta}|^{2}+\left|\nabla_{x} \vartheta\right|^{2}\right] \mathrm{d} x d s \\
& \leq C\left(e_{0}^{2}+A^{4 / 3}+A^{3}\right)+C \int_{0}^{t} \phi^{5 / 3}\|\dot{\vartheta}\|^{4 / 3}\left|\nabla_{x} \dot{\vartheta}\right|^{2} d s+C A \int_{0}^{t}|\Delta \vartheta|^{2} d s \\
& \leq C\left(e_{0}^{2}+A^{4 / 3}+A^{3}\right) .
\end{aligned}
$$

Inserting into (3.45) we get (3.43).

3. Using the energy equation (1.5), (3.1) and (3.43), we get (3.44). 
Lemma 3.10. There is a positive constant $\mathcal{C}$ such that the following inequality holds

$$
A(T) \leq \mathcal{C}\left(e_{0}^{2}+A^{4 / 3}(T)+A^{3}(T)\right) .
$$

Proof. The proof follows by combining the definition (2.20) of $A(T)$ and the estimates in Lemmas 3.2, 3.6, 3.7, 3.8 and 3.9.

\section{Proof of Proposition 2.3}

As one checks that $A(0) \leq 2 \mathcal{C} e_{0}^{2}$, and due to the continuity of $T \rightarrow A(T)$, it follows from Lemma 3.10 that the inequality

$$
A(T) \leq 2 \mathcal{C} e_{0}^{2}
$$

also holds for any $T>0$, provided $e_{0}$ is small enough, say $e_{0}<\eta(\mathcal{C})$.

Using Lemma 3.2 and (4.1) we get

$$
\begin{aligned}
& \sup _{t \geq 0}\left\{\|\varrho-\bar{\varrho}\|_{L^{\infty}}+\phi(t)\|\vartheta-\bar{\vartheta}\|_{L^{\infty}}+\left\|E^{R}-\bar{E}\right\|_{L^{\infty}}\right\} \\
& \leq C\left(e_{0}+A^{1 / 2}(T)\right)+C \sup _{t \geq 0}\left\{\phi(t)\|\vartheta-\bar{\vartheta}\|_{L^{\infty}}+\left\|\nabla_{x} \vartheta\right\|_{L^{\infty}}+\|\Delta \vartheta\|_{L^{\infty}}\right\} \\
& \leq C\left(e_{0}+A^{1 / 2}(T)\right) \leq \tilde{\eta} e_{0} \leq \frac{1}{3} \min \{|\overline{\vec{u}}|, \bar{\vartheta}\}
\end{aligned}
$$

provided that

$$
e_{0} \leq \min \left\{\eta(\mathcal{C}), \frac{|\overline{\vec{u}}|}{3 \tilde{\eta}}, \frac{\bar{\vartheta}}{3 \tilde{\eta}}\right\}:=\varepsilon .
$$

So for data such that $e_{0} \leq \varepsilon$ and provided that (3.1) holds, estimate (4.2) is valid. Since (4.2) is true for $t=0$ and holds also for any $T \geq 0$ after continuity of $\varrho$ and $\vartheta$, one sees that (4.1) is also valid for any $T \geq 0$. Then provided that $e_{0} \leq \varepsilon$, one concludes that (4.1) and (4.2) are valid for any $T \geq 0$.

Now using the Sobolev imbedding theorem, we deduce that

$$
\phi^{2}(t)\left(\left\|\nabla_{x} \vec{u}\right\|_{L^{4}}^{4}+\|\vec{u}\|_{L^{\infty}}^{4}\right) \leq \mathcal{C} e_{0}^{2} \text { for any } t \in[0, T],
$$

and for $T>1$

$$
\left.\|F\|_{L^{\infty}}^{10 / 3}+\| \vartheta-\bar{\vartheta}\right\} \|_{L^{\infty}}^{10 / 3} \leq C\left(\|\dot{\vec{u}}\|^{2}+\left\|\nabla_{x} \dot{\vec{u}}\right\|^{2}+\left\|\nabla_{x} \vartheta\right\|^{2}+\|\Delta \vartheta\|^{2}\right) .
$$


So, multiplying (3.12) by $\operatorname{sgn}(\varrho-\bar{\varrho})|\varrho-\bar{\varrho}|^{7 / 3}$ and integrating by parts on $[1, T] \times$ $\mathbb{R}^{3}$, we find

$$
\begin{aligned}
|\varrho(T)-\bar{\varrho}|_{L^{\infty}}^{10 / 3} \leq & e^{\gamma(1-T)}|\varrho(1)-\bar{\varrho}|_{L^{\infty}}^{10 / 3} \\
& \left.+C \int_{1}^{T} e^{-\gamma(T-t)}\left(\|F\|_{L^{\infty}}^{10 / 3}+\| \vartheta-\bar{\vartheta}\right\} \|_{L^{\infty}}^{10 / 3}\right) d t \\
\leq & C e^{-\gamma \frac{T}{2}}+C \int_{\frac{T}{2}}^{T}\left(\|\dot{\vec{u}}\|^{2}+\left\|\nabla_{x} \dot{\vec{u}}\right\|^{2}+\left\|\nabla_{x} \vartheta\right\|^{2}+\|\Delta \vartheta\|^{2}\right) d t
\end{aligned}
$$

with $\gamma=\frac{\bar{\varrho} p_{\varrho}(\bar{\varrho}, \bar{\vartheta})}{2(\lambda+2 \mu)}$, which shows that $\|\varrho(T)-\bar{\varrho}\|_{L^{\infty}} \rightarrow 0$ when $T \rightarrow \infty$. As one checks that $t \rightarrow\left\|\nabla_{x} \vec{u}\right\|^{2}+\left\|\nabla_{x} \vartheta\right\|^{2}$ and $t \rightarrow \frac{d}{d t}\left(\left\|\nabla_{x} \vec{u}\right\|^{2}+\left\|\nabla_{x} \vartheta\right\|^{2}\right)$ are in $L^{1}(1, \infty)$, we also see that $\left\|\nabla_{x} \vec{u}(T)\right\|^{2} \rightarrow 0$ when $T \rightarrow \infty$ and also that $\left\|\nabla_{x} \vartheta(T)\right\|^{2} \rightarrow 0$ when $T \rightarrow \infty$. As $t \rightarrow\left(\|\vec{u}\|,\|\varrho-\bar{\varrho}\|,\|\vec{u}\|_{L^{\infty}},\|\varrho-\bar{\varrho}\|_{L^{\infty}}\right)$ are uniformly bounded for $t \geq 1$, we conclude that $\|\vec{u}\|_{L^{q}} \rightarrow 0$ when $T \rightarrow \infty$ and $\|\varrho-\bar{\varrho}\|_{L^{q}} \rightarrow 0$ when $T \rightarrow \infty$.

Finally, multiplying (1.6) by $I-\bar{I}$ and integrating by parts on $[1, T] \times \mathbb{R}^{3} \times$ $\mathcal{S}^{2} \times \mathbb{R}_{+}$, we find

$$
\begin{aligned}
& \|I-\bar{I}\|^{2} \leq e^{\delta(1-T)}\|I(1)-\bar{I}\|^{2} \\
& +C \int_{1}^{T} e^{-\delta(T-t)}\left\|\sigma_{a}\right\|_{L^{1}\left(\mathbb{R}_{+}, d \nu\right) \times L^{\infty}\left(\mathbb{R}^{3}, d x\right)}\left\|\partial_{\vartheta} B\right\|_{L^{1}\left(\mathbb{R}_{+}, d \nu\right) \times L^{\infty}\left(\mathbb{R}^{3}, d x\right)}\|\vartheta-\bar{\vartheta}\|^{2} d t,
\end{aligned}
$$

with $\delta=c\left(\sigma_{a}+\sigma_{s}\right)$, which shows that $\|I(T)-\bar{I}\| \rightarrow 0$ when $T \rightarrow \infty$, and consequently by integrating on $\mathbb{R}^{3}\left\|E^{R}(T)-\bar{E}\right\| \rightarrow 0$ when $T \rightarrow \infty$, which ends the proof of Proposition 2.3.

\section{Proof of Theorem 2.2}

In this section, we suppose that the initial energy is close to equilibrium: we assume that $e_{0} \leq \varepsilon$ where the $\varepsilon$ is the same as in the previous section. Following [25], we define

$$
\begin{aligned}
B(T)= & \sup _{t \in[0, T]}\left(\left\|\nabla_{x} \varrho\right\|^{2}+\left\|\nabla_{x} \varrho\right\|_{L^{\alpha}}^{\alpha}\right)(t) \\
& +\int_{0}^{\phi(T)}\left(\|\vartheta-\bar{\vartheta}\|_{L^{\infty}}^{2}+\left\|E^{R}-\overline{E^{R}}\right\|_{L^{\infty}}^{2}\right) d s \\
& +\left[\int_{0}^{\phi(T)}\left(\left\|\nabla_{x} \varrho\right\|+\left\|\nabla_{x} \varrho\right\|_{L^{\alpha}}^{\alpha}+\left\|\nabla_{x} \vec{u}\right\|_{L^{\infty}}\right) d s\right]^{2} \\
& +\int_{1}^{\max \{1, T\}}\left(\left\|\nabla_{x} \varrho\right\|^{2}+\left\|\nabla_{x} \varrho\right\|_{L^{\alpha}}^{2}\right) d s .
\end{aligned}
$$


First note that, applying $\partial_{j}$ to equation (3.12), we get

$$
\begin{aligned}
& (\lambda+2 \mu) \frac{d}{d t} \partial_{j} \varrho+\varrho \partial_{\varrho} p(\bar{\varrho}, \bar{\vartheta}) \partial_{j} \varrho \\
& =-\varrho \partial_{j} F-F \partial_{j} \varrho-\left[\partial_{\varrho} p(\varrho, \vartheta)-\partial_{\varrho} p(\bar{\varrho}, \bar{\vartheta})\right] \partial_{j} \varrho-\varrho \partial_{\vartheta} p \partial_{j} \vartheta-(p-\bar{p}) \partial_{j} \varrho \\
& \quad-(\lambda+2 \mu) \partial_{j} u_{k} \partial_{k} \varrho .
\end{aligned}
$$

Multiplying, for $p \geq 2$ and $m \in \mathbb{N}$, this identity by $\operatorname{sgn}\left(\partial_{j} \varrho\right) \varrho\left|\partial_{j} \varrho\right|^{p-1}\left\|\varrho^{1 / p} \partial_{j} \varrho\right\|_{L^{p}}^{m}$, integrating on $\mathbb{R}^{3}$ and using Hôlder's inequality, and the definition of $F$ we find

$$
\begin{aligned}
& (\lambda+2 \mu) \frac{d}{d t}\left\|\varrho^{1 / p} \partial_{j} \varrho\right\|_{L^{p}}^{m}+\partial_{\varrho} p(\bar{\varrho}, \bar{\vartheta})\left\|\varrho^{1 / p} \partial_{j} \varrho\right\|_{L^{p}}^{m} \\
& \leq C\left\{\|\varrho-\bar{\varrho}\|_{L^{\infty}}+\left\|\nabla_{x} \vec{u}\right\|_{L^{\infty}}+\|\vartheta-\bar{\vartheta}\|_{L^{\infty}}+\|F\|_{L^{\infty}}\right\}\left\|\partial_{j} \varrho\right\|_{L^{p}}^{m} \\
& \quad+C\left(\|\dot{\vec{u}}\|_{L^{p}}^{m}+\left\|\partial_{j} \vartheta\right\|_{L^{p}}^{m}+\|\vartheta-\bar{\vartheta}\|_{L^{p}}^{m}+\|I-\bar{I}\|_{L^{p}}^{m}\right) .
\end{aligned}
$$

Consider the case $T \leq 1$. Taking $m=1$ and successively $p=2$ and $p=\alpha$, summing, integrating over $[0, t]$ for $t \leq T \leq 1$ and using the definition of $B(T)$, we have

$$
\begin{aligned}
& \sup _{t \in[0, T]}\left(\left\|\nabla_{x} \varrho\right\|+\left\|\nabla_{x} \varrho\right\|_{L^{\alpha}}\right)+\int_{0}^{T}\left(\left\|\nabla_{x} \varrho\right\|+\left\|\nabla_{x} \varrho\right\|_{L^{\alpha}}\right) d s \\
& \leq C\left(E_{0}+B\right)+C \int_{0}^{T}\left(\left\|\nabla_{x} \vartheta\right\|+\|\Delta \vartheta\|\right) d s \leq C\left(E_{0}+B\right) .
\end{aligned}
$$

When $T \geq 1$, using equation (1.5), estimates (4.1) and (4.2), and the elliptic regularity of $\mu \Delta+(\mu+\lambda) \nabla_{x} \operatorname{div}_{x}$, we get

$$
\begin{aligned}
\left\|\nabla_{x} \vec{u}\right\|_{L^{\infty}} \leq & C\left(\left\|\nabla_{x} \vec{u}\right\|+\left\|\nabla_{x}^{2} \vec{u}\right\|+\left\|\nabla_{x}^{2} \vec{u}\right\|_{L^{\alpha}}\right) \\
\leq & C E_{0}+C\left(\|\dot{\vec{u}}\|+\|\vartheta-\bar{\vartheta}\|+\|I-\bar{I}\|+\left\|\nabla_{x} p\right\|+\left\|\vec{S}_{F}\right\|\right) \\
& +C\left(\|\dot{\vec{u}}\|_{L^{\alpha}}+\|\vartheta-\bar{\vartheta}\|_{L^{\alpha}}+\|I-\bar{I}\|_{L^{\alpha}}+\left\|\nabla_{x} p\right\|_{L^{\alpha}}+\left\|\vec{S}_{F}\right\|_{L^{\alpha}}\right) \\
\leq & C\left(E_{0}+B^{1 / 2}+\left\|\nabla_{x} \dot{\vec{u}}\right\|\right) .
\end{aligned}
$$

Taking $m=1$ and successively $p=2$ and $p=\alpha$ in (5.1), summing, integrating over [1,t] for $t \leq T \leq 1$ and using the definition of $B(T)$ together with bounds 
(5.2) and (5.3), we now have

$$
\begin{aligned}
& \sup _{t \in[1, T]}\left(\left\|\nabla_{x} \varrho\right\|+\left\|\nabla_{x} \varrho\right\|_{L^{\alpha}}\right)+\int_{1}^{T}\left(\left\|\nabla_{x} \varrho\right\|+\left\|\nabla_{x} \varrho\right\|_{L^{\alpha}}\right) d s \\
& \leq C\left(\left\|\nabla_{x} \varrho\right\|+\left\|\nabla_{x} \varrho\right\|_{L^{\alpha}}\right)(1) \\
& \quad+C \int_{1}^{T}\left\{\left(E_{0}+B^{1 / 2}+\left\|\nabla_{x} \dot{\vec{u}}\right\|+\left\|\nabla_{x} \vartheta\right\|+\left\|\nabla_{x} I\right\|\right)\left(\left\|\nabla_{x} \varrho\right\|^{2}+\left\|\nabla_{x} \varrho\right\|_{L^{\alpha}}^{2}\right)\right. \\
& \left.\quad+\left\|\nabla_{x} \dot{\vec{u}}\right\|^{2}+\left\|\nabla_{x} \vartheta\right\|^{2}+\|\dot{\vec{u}}\|_{L^{\alpha}}^{2}+\left\|\nabla_{x} \vartheta\right\|_{L^{\alpha}}^{2}\right\} d s \\
& \leq C\left(E_{0}^{2}+B^{3 / 2}(T)+B^{2}(T)\right) .
\end{aligned}
$$

Taking (5.2) into account, we finally get

$$
\begin{aligned}
& \sup _{0 \in[1, T]}\left(\left\|\nabla_{x} \varrho\right\|+\left\|\nabla_{x} \varrho\right\|_{L^{\alpha}}\right)+\left\{\int_{1}^{\min \{1, T\}}\left(\left\|\nabla_{x} \varrho\right\|+\left\|\nabla_{x} \varrho\right\|_{L^{\alpha}}\right) d s\right\}^{2} \\
& +\int_{1}^{\max \{1, T\}}\left(\left\|\nabla_{x} \varrho\right\|+\left\|\nabla_{x} \varrho\right\|_{L^{\alpha}}\right) d s \leq C\left(E_{0}^{2}+B^{3 / 2}(T)+B^{2}(T)\right),
\end{aligned}
$$

for any $T \geq 0$.

From the Sobolev imbedding theorem, we have

$$
\int_{1}^{\min \{1, T\}}\|\vartheta-\bar{\vartheta}\|_{L^{\infty}}^{2} d s \leq C E_{0}^{2},
$$

and by the same arguments leading to (5.2), one finds

$$
\int_{1}^{\min \{1, T\}}\left\|\nabla_{x} \vec{u}\right\|_{L^{\infty}}^{2} d s \leq C\left(E_{0}+B^{3 / 4}(T)+B(T)\right) .
$$

Combining (5.4), (5.5) and (5.6) to (4.2), we get

$$
B(T) \leq C\left(E_{0}^{2}+B^{3 / 2}(T)+B^{2}(T)\right) .
$$

As $B(0) \leq 2 C E_{0}^{2}$, we finally obtain:

Lemma 5.1. The following bound holds

$$
B(T)<2 C E_{0}^{2}
$$

provided that $E_{0} \leq \min \left\{\frac{1}{8 C^{2}}, \varepsilon\right\}:=\varepsilon^{\prime}$, where $\varepsilon$ is the small number in (4.2).

Now after Proposition 2.3 and Lemmas 5.2 and 5.1, using (4.1), (4.2) and (4.3) and following straightfully the estimates of Jiang for higher order derivatives, we obtain the final following a-priori estimates: 
Proposition 5.2. Let $T_{*}$ be a fixed number such that $0<T_{*}<\min \{1, T\}$. Under the hypotheses of Theorem 2.2, the following estimates hold:

1. $\underline{\rho} \leq \varrho(x, t) \leq \bar{\rho}, \quad \underline{\theta} \leq \vartheta(x, t) \leq \bar{\theta}$ for all $(x, t) \in \mathbb{R}^{3} \times\left[T_{*}, T\right]$,

2. $\sup _{\left[T_{*}, T\right]}\left(\|\varrho-\bar{\varrho}\|_{H^{3}}+\|\vec{u}\|_{H^{3}}+\|\vartheta-\bar{\vartheta}\|_{H^{3}}+\left\|E^{R}-\overline{E^{R}}\right\|_{H^{3}}\right.$

$$
\left.+\left\|\partial_{t} \varrho\right\|_{H^{2}}+\left\|\partial_{t} E^{R}\right\|_{H^{2}}+\left\|\partial_{t} \vec{u}\right\|_{H^{1}}+\left\|\partial_{t} \vartheta\right\|_{H^{1}}\right) \leq K,
$$

where $K$ depends on physical constants and of $\underline{\rho}, \bar{\rho}, \underline{\theta}$ and $\bar{\theta}$,

3. $\begin{aligned} \int_{T_{*}}^{T} & \left\{\left\|\nabla_{x} \varrho\right\|_{H^{2}}^{2}+\left\|\nabla_{x} \vec{u}\right\|_{H^{2}}^{2}+\left\|\nabla_{x} \vartheta\right\|_{H^{2}}^{2}+\left\|\nabla_{x} E^{R}\right\|_{H^{2}}^{2}\right. \\ & \left.+\left\|\partial_{t} \varrho\right\|_{H^{2}}+\left\|\partial_{t} E^{R}\right\|_{H^{2}}+\left\|\partial_{t} \vec{u}\right\|_{H^{1}}+\left\|\partial_{t} \vartheta\right\|_{H^{1}}\right\} d s \leq K,\end{aligned}$

provided that $E_{0} \leq \varepsilon^{\prime}$, where $\varepsilon^{\prime}$ is the small number in Lemma 5.1.

Now we have the following local existence theorem (proved in the Appendix):

Theorem 5.3. Suppose that $\|\varrho-\bar{\varrho}\|_{H^{3}}+\|\vec{u}\|_{H^{3}}+\|\vartheta-\bar{\vartheta}\|_{H^{3}}+\left\|E^{R}-\overline{E^{R}}\right\|_{H^{3}}<\infty$

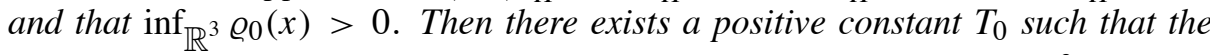
Cauchy problem (1.3-2.1) has a unique solution $(\varrho, \vec{u}, \vartheta)$ on $\left[0, T_{0}\right] \times \mathbb{R}^{3}$ and I on $\left[0, T_{0}\right] \times \mathbb{R}^{3} \times \mathcal{S}^{2} \times \mathbb{R}_{+}$, satisfying:

$$
\begin{aligned}
& \left(\varrho, \vec{u}, \vartheta, E^{R}\right) \in C^{0}\left(0, T_{0} ; H^{3}\right), \\
& \left(\partial_{t} \varrho, \partial_{t} E^{R}\right) \in C^{0}\left(0, T_{0} ; H^{2}\right), \\
& (\partial \vec{u}, \partial \vartheta) \in C^{0}\left(0, T_{0} ; H^{1}\right), \\
& \partial_{t} \varrho \in C^{0}\left(0, T_{0} ; H^{2}\right), \\
& (\vec{u}, \vartheta-\bar{\vartheta}) \in L^{2}\left(0, T_{0} ; H^{4}\right) .
\end{aligned}
$$

2. For some $M>0$ independent of $T_{*}$

$$
\begin{aligned}
& \varrho(x, t) \geq \frac{1}{2} \inf _{\mathbb{R}^{3}} \varrho_{0}(x), \text { on }\left[0, T_{0}\right] \times \mathbb{R}^{3}, \\
& \sup _{\left[T_{*}, T\right]}\left\{\|\varrho-\bar{\varrho}\|_{H^{3}}+\|\vec{u}\|_{H^{3}}+\|\vartheta-\bar{\vartheta}\|_{H^{3}}+\left\|E^{R}-\overline{E^{R}}\right\|_{H^{3}}\right\} \\
& \quad+\int_{T_{*}}^{T}\left\{\left\|\nabla_{x} \vec{u}\right\|_{H^{3}}^{2}+\left\|\nabla_{x} \vartheta\right\|_{H^{3}}^{2}+\left\|\nabla_{x} E^{R}\right\|_{H^{3}}^{2}\right\} d s \\
& \leq M\left(\left\|\varrho_{0}-\bar{\varrho}\right\|_{H^{3}}+\left\|\vec{u}_{0}\right\|_{H^{3}}+\left\|\vartheta_{0}-\bar{\vartheta}\right\|_{H^{3}}+\left\|E_{0}^{R}-\overline{E^{R}}\right\|_{H^{3}}\right) .
\end{aligned}
$$

Moreover if $\inf _{\mathbb{R}^{3}} \vartheta_{0}(x)>0$, then $\vartheta(x, t) \geq 0$ on $\left[0, T_{0}\right] \times \mathbb{R}^{3}$. 
Using this local result, we can end the proof of Theorem 2.2. Under the conditions of Theorem 2.2, the Cauchy problem (1.3-2.1) has a unique solution $(\varrho, \vec{u}, \vartheta)$ on $\left[0, T_{0}\right] \times \mathbb{R}^{3}$ and $I$ on $\left[0, T_{0}\right] \times \mathbb{R}^{3} \times \mathcal{S}^{2} \times \mathbb{R}_{+}$after Theorem 5.3. Using the a priori estimates of Proposition 5.2, this solution can be continued globally in time and the estimates of Proposition 5.2 are valid for any $T \geq 0$.

The asymptotic behaviour follows by observing that after (2.14)

$$
\begin{aligned}
& \int_{1}^{\infty}\left|\frac{d}{d t}\left\{\|\Delta \varrho\|^{2}+\|\Delta \vec{u}\|^{2}+\|\Delta \vartheta\|^{2}+\left\|\Delta E^{R}\right\|^{2}\right\}\right| d s \\
& \leq \int_{1}^{\infty}\left\{\left\|\Delta \partial_{t} \varrho\right\|^{2}+\left\|\Delta \partial_{t} \vec{u}\right\|^{2}+\left\|\Delta \partial_{t} \vartheta\right\|^{2}+\left\|\Delta \partial_{t} E^{R}\right\|^{2}\right. \\
& \left.+\|\Delta \varrho\|^{2}+\|\Delta \vec{u}\|^{2}+\|\Delta \vartheta\|^{2}+\left\|\Delta E^{R}\right\|^{2}\right\} d s<\infty .
\end{aligned}
$$

Combining this to (5.10), we see that $\|\Delta \varrho\|^{2}+\|\Delta \vec{u}\|^{2}+\|\Delta \vartheta\|^{2}+\left\|\Delta E^{R}\right\|^{2} \rightarrow 0$ as $t \rightarrow \infty$. Using now (5.8) and Gagliardo-Nirenberg's inequality, we conclude that

$$
\begin{gathered}
\|\varrho-\bar{\varrho}\|_{L^{\infty}}+\|\vec{u}\|_{L^{\infty}}+\|\vartheta-\bar{\vartheta}\|_{L^{\infty}}+\left\|E^{R}-\overline{E^{R}}\right\|_{L^{\infty}} \\
\leq C\left(\|\varrho-\bar{\varrho}\|+\|\vec{u}\|+\|\vartheta-\bar{\vartheta}\|+\left\|E^{R}-\overline{E^{R}}\right\|\right)^{1 / 4} \\
\quad \times\left(\|\Delta \varrho\|+\|\Delta \vec{u}\|+\|\Delta \vartheta\|+\left\|\Delta E^{R}\right\|\right)^{3 / 4} \rightarrow 0,
\end{gathered}
$$

as $t \rightarrow \infty$, which ends the proof of Theorem 2.2.

\section{A. Appendix: Proof of Theorem 5.3}

In fact a proof can be derived in various ways from the works of Nash [41], Matsumura-Nishida [37], or Solonnikov-Kazhikov [44] (among others) and we only sketch the extension to the radiative case. Let us mention that the existence local in time for the inviscid case was proved in $[33,48]$. It will be convenient to switch to Lagrangian variables. Conserving the same notation for the variables and the unknowns, the problem rewrites as

$$
\left\{\begin{array}{l}
\partial_{t} \varrho+\varrho \operatorname{div} \vec{u}=0, \\
\varrho \partial_{t} \vec{u}+\nabla p-\nabla(\lambda \operatorname{div} \vec{u})-2 \nabla \cdot(\mu \mathbb{D})=-\vec{S}_{F}, \\
\varrho e_{\vartheta} \partial_{t} \vartheta+\vartheta p_{\vartheta} \operatorname{div} \vec{u}+\operatorname{div}(\kappa \nabla \vartheta)-2 \mu \mathbb{D}: \mathbb{D}-\lambda(\operatorname{div} \vec{u})^{2}=-S_{E}, \\
\partial_{t} I+(c \vec{\omega}-\vec{u}) \cdot \nabla I=c S, \\
(\varrho(0, x), \vec{u}(0, x), \vartheta(0, x), I(0, x, \vec{\omega}, v))=\left(\varrho_{0}(x), \vec{u}_{0}(x), \vartheta_{0}(x), I_{0}(x, \vec{\omega}, v)\right),
\end{array}\right.
$$

for $(t, x, \vec{\omega}, v) \in(0, T) \times \mathbb{R}^{3} \times \mathcal{S}^{2} \times \mathbb{R}_{+}$, where $\nabla$ and div are the new operators. 
We consider the auxiliary linear Cauchy problem

$$
\left\{\begin{array}{l}
\partial_{t} \varrho+\varrho^{\prime} \operatorname{div} \vec{u}=0, \\
\varrho^{\prime} \partial_{t} \vec{u}+p_{\varrho}^{\prime} \nabla \varrho+p_{\vartheta}^{\prime} \nabla \vartheta-\nabla(\lambda \operatorname{div} \vec{u})-2 \nabla \cdot(\mu \mathbb{D})=-\vec{S}_{F}^{\prime}, \\
\varrho^{\prime} e_{\vartheta}^{\prime} \partial_{t} \vartheta+\vartheta^{\prime} p_{\vartheta}^{\prime} \operatorname{div} \vec{u}+\operatorname{div}(\kappa \nabla \vartheta)-2 \mu \mathbb{D}^{\prime}: \mathbb{D}-\lambda \operatorname{div} \vec{u}^{\prime} \operatorname{div} \vec{u}=-S_{E}^{\prime}, \\
\partial_{t} I+\left(c \vec{\omega}-\vec{u}^{\prime}\right) \cdot \nabla I=c S^{\prime}, \\
(\varrho(0, x), \vec{u}(0, x), \vartheta(0, x), I(0, x, \vec{\omega}, \nu))=\left(\varrho_{0}(x), \vec{u}_{0}(x), \vartheta_{0}(x), I_{0}(x, \vec{\omega}, \nu)\right),
\end{array}\right.
$$

where

$$
\begin{aligned}
& p_{\varrho}^{\prime}=p_{\varrho}\left(\varrho^{\prime}, \vartheta^{\prime}\right), \quad p_{\vartheta}^{\prime}=p_{\vartheta}\left(\varrho^{\prime}, \vartheta^{\prime}\right), \quad e_{\vartheta}^{\prime}=e_{\vartheta}\left(\varrho^{\prime}, \vartheta^{\prime}\right) \\
& S^{\prime}=\sigma_{a}\left(\vartheta^{\prime}, v\right)\left(B\left(\vartheta^{\prime}, v\right)-I\right)+\sigma_{s}\left(\vartheta^{\prime}, v\right)\left(\frac{1}{4 \pi} \int_{\mathcal{S}^{2}} I(\cdot, \vec{\omega}) \mathrm{d} \vec{\omega}-I\right),
\end{aligned}
$$

and

$$
\vec{S}_{F}=\int_{\mathcal{S}^{2}} \vec{\omega} S^{\prime} d \vec{\omega} d \nu, \quad S_{E}^{\prime}=\int_{\mathcal{S}^{2}} S^{\prime} d \vec{\omega} d \nu
$$

Clearly, the first and last equations in (A.2) are hyperbolic equations which can be solved explicitly through the method of characteristics, and system (A.2) can be considered as a linear parabolic problem for the pair $U:=(\vec{u}, \vartheta)$ and rewrites as

$$
\left\{\begin{array}{l}
\mathcal{L}\left(U^{\prime}\right) U=h\left(U^{\prime}\right) \\
\left.U\right|_{t=0}=U_{0} \equiv\left(\vec{u}_{0}, \vartheta_{0}\right)
\end{array}\right.
$$

The initial problem corresponds clearly to $U^{\prime}=U$

$$
\left\{\begin{array}{l}
\mathcal{L}(U) U=h(U) \\
\left.U\right|_{t=0}=U_{0} \equiv\left(\vec{u}_{0}, \vartheta_{0}\right)
\end{array}\right.
$$

and we can reduce this nonlinear problem to a fixed point problem in the Banach space $\left(C^{2+\alpha, 1+\frac{\alpha}{2}}\left(\Sigma_{\tau}\right),\|\cdot\|_{\Sigma_{\tau}}^{(2+\alpha)}\right)$ where $\Sigma_{\tau}$ is the domain $\Sigma_{\tau}=\{(t, x) \in[0, \tau] \times$ $\left.\mathbb{R}^{3}\right\}$, where $\tau>0$ is small enough. So we define the vector $U_{1}$ as the solution of the Cauchy problem in $\Sigma_{\tau}$

$$
\left\{\begin{array}{l}
\mathcal{L}\left(U_{0}\right) U_{1}=h\left(U_{0}\right) \\
\left.U_{1}\right|_{t=0}=U_{0} \equiv\left(\vec{u}_{0}, \vartheta_{0}\right)
\end{array}\right.
$$

Introducing the difference $V=U_{1}-U_{0}$ and subtracting (A.4) to (A.5), we get

$$
\left\{\begin{aligned}
\mathcal{L}\left(U_{1}\right) V= & {\left[\mathcal{L}\left(U_{1}\right)-\mathcal{L}\left(U_{1}+V\right)\right]\left(U_{1}+V\right)+h\left(U_{1}+V\right)-h\left(U_{1}\right) } \\
& +\left[\mathcal{L}\left(U_{0}\right)-\mathcal{L}\left(U_{1}\right)\right] U_{1}+h\left(U_{1}\right)-h\left(U_{0}\right) \equiv \mathcal{B}_{\tau} V, \\
\left.V\right|_{t=0}= & 0 .
\end{aligned}\right.
$$


This problem is equivalent to the nonlinear equation

$$
\begin{aligned}
V= & \mathcal{R}_{\tau}\left\{\left[\mathcal{L}\left(U_{1}\right)-\mathcal{L}\left(U_{1}+V\right)\right]\left(U_{1}+V\right)+h\left(U_{1}+V\right)-h\left(U_{1}\right)\right\} \\
& +\mathcal{R}_{\tau}\left\{\left[\mathcal{L}\left(U_{0}\right)-\mathcal{L}\left(U_{1}\right)\right] U_{1}+h\left(U_{1}\right)-h\left(U_{0}\right) \equiv \mathcal{B}_{\tau} V\right\} \equiv \mathcal{B}_{\tau} V,
\end{aligned}
$$

where $\mathcal{R}_{\tau}$ is a linear operator defined on the Banach space $\left(C^{\alpha, \frac{\alpha}{2}}\left(\Sigma_{\tau}\right),\|\cdot\|_{\Sigma_{\tau}}^{(\alpha)}\right)$ by considering $W=\mathcal{R}_{\tau} f$ as the solution of the Cauchy problem

$$
\left\{\begin{array}{c}
\mathcal{L}\left(U_{1}\right) W=f \\
\left.W\right|_{t=0}=0
\end{array}\right.
$$

Choosing $\tau$ sufficiently small, such that

$$
\left(\tau^{1 / 2-\alpha / 2}\right)\|U\|_{\Sigma_{\tau}}^{(2+\alpha)} \leq \delta
$$

holds for a small $\delta$, the vector $h(U)$ and the coefficients of the operator $\mathcal{L}(U)$ are smooth functions of their arguments. Then, if $U_{1}, U_{1}+V$ and $U_{1}+W$ satisfy (A.9) and $\left.V\right|_{t=0}=\left.W\right|_{t=0}=0$, then

$$
\left\|\mathcal{B}_{\tau} V\right\|_{\Sigma_{\tau}}^{(2+\alpha)} \leq \tau^{\gamma}\left\|\mathcal{R}_{\tau}\right\|\left(C_{1}\|V\|_{\Sigma_{\tau}}^{(2+\alpha)}+C_{2}\left\|U_{0}-U_{1}\right\|_{\Sigma_{\tau}}^{(2+\alpha)}\right),
$$

and

$$
\left\|\mathcal{B}_{\tau} V-\mathcal{B}_{\tau} W\right\|_{\Sigma_{\tau}}^{(2+\alpha)} \leq C_{3} \tau^{\gamma}\left\|\mathcal{R}_{\tau}\right\|\|V-W\|_{\Sigma_{\tau}}^{(2+\alpha)},
$$

for a $\gamma>0$ and constants $C_{j}$ independent of $\tau, V$ or $W$.

Choosing now the positive numbers $T_{*}$ and $\eta>0$ such that

$$
\begin{gathered}
\left(T_{*}+T_{*}^{1 / 2-\alpha / 2}\right)\left\|U_{0}\right\|_{\Sigma_{T_{*}}}^{(2+\alpha)} \leq \delta, \quad\left(T_{*}+T_{*}^{1 / 2-\alpha / 2}\right)\left\|U_{1}\right\|_{\Sigma_{T_{*}}}^{(2+\alpha)} \leq \frac{\delta}{2}, \\
C_{3} T_{*}^{\gamma}\left\|\mathcal{R}_{T_{*}}\right\|<1, \quad C_{1} T_{*}^{\gamma}\left\|\mathcal{R}_{T_{*}}\right\|<\frac{1}{2},
\end{gathered}
$$

and

$$
2 C_{2} T_{*}^{\gamma}\left\|\mathcal{R}_{T_{*}}\right\|\left\|U_{0}-U_{1}\right\|_{\Sigma_{\tau}}^{(2+\alpha)} \leq \eta \leq \frac{\delta}{2\left(T_{*}+T_{*}^{1 / 2-\alpha / 2}\right)},
$$

and denoting by $\mathcal{K}_{\eta}$ the set

$$
\mathcal{K}_{\eta}=\left\{V \in C^{2+\alpha, 1+\frac{\alpha}{2}}\left(\Sigma_{\tau}\right), V(x, 0)=0,\|V\|_{\Sigma_{\tau}}^{(2+\alpha)} \leq \eta\right\},
$$

we observe after (A.10)-(A.14) that $U_{0}$ and $U_{1}+V$ together with any element of $\mathcal{K}_{\eta}$ satisfy (A.9) and that the norm $\left\|\mathcal{R}_{T_{*}}\right\|$ is bounded. Moreover $\mathcal{B}_{T_{*}}$ is a contraction from $\mathcal{K}_{\eta}$ into itself then equation (A.7) has a unique solution in the Hölder framework. Passing to the Sobolev setting is routine and we omit this step. This ends the proof of Theorem 5.3. 


\section{References}

[1] A. A. Amosov, Well-posedness "in the large" initial and boundary-value problems for the system of dynamical equations of a viscous radiating gas, Sov. Physics Dokl. 30 (1985), 129-131.

[2] R. Balian, "From Microphysics to Macrophysics. Methods and Applications of Statistical Physics", Vol. II, Springer Verlag, Berlin, Heidelberg, New York, 1992.

[3] C. BARdos, F. Golse, B. PERTHAME and R. SENTIS, The nonaccretive radiative transfer equations: Existence of solutions and Rosseland approximation, J. Funct. Anal. 77 (1988), 434-460.

[4] F. BERTHELIN and S. JUNCA, Averaging lemmas with a force term in the transport equation, J. Math. Pures Appl. (9) 93 (2010), 113-131.

[5] N. Bournaveas and B. PERTHAME, Averages over spheres for kinetic transport equations; hyperbolic Sobolev spaces and Strichartz inequalities, J. Math. Pures Appl. 80 (9) (2001), 517-534.

[6] C. BUET and B. DESPRÉs, Asymptotic analysis of fluid models for the coupling of radiation and hydrodynamics, J. Quant. Spectroscopy Rad. Transf. 85 (2004), 385-480.

[7] BUI AN TON, On the initial boundary-value problem for viscous heat-conducting compressible fluids, Kodai Math. J. 4 (1981), 97-128.

[8] S. ChandRaseKhar, "Radiative transfer", Dover Publications, New York, 1960.

[9] R. J. DiPERNA and P.-L. LIONS, Ordinary differential equations, transport theory and Sobolev spaces, Invent. Math. 98 (1989), 511-547.

[10] B. Dubroca and J.-L. Feugeas, Etude théorique et numérique d'une hiérarchie de modéles aux moments pour le transfert radiatif, C. R. Math. Acad. Sci. Paris 329 (1999), 915-920.

[11] B. Dubroca, M. Seaïd and J.-L. Feugeas, A consistent approach for the coupling of radiation and hydrodynamics at low Mach number, J. Comput. Phys. 225 (2007), 10391065.

[12] B. DuCOMET, E. FeIREISL and Š. NeČASOVÁ, On a model of radiation hydrodynamics, Ann. Inst. H. Poincaré Anal. Non Linéaire 28 (2011), 797-812.

[13] B. DUCOMET and Š. NEČASOVÁ, Global existence of solutions for the one-dimensional motions of a compressible gas with radiation: an "infrarelativistic model", Nonlinear Anal. 72 (2010), 3258-3274.

[14] B. DUCOMET and Š. NEČASOVÁ, Global weak solutions to the 1D compressible NavierStokes equations with radiation, Commun. Math. Anal. 8 (2010), 23-65.

[15] B. DUCOMET and Š. NEČASOVÁ, Large-time behavior of the motion of a viscous heatconducting one-dimensional gas coupled to radiation, Ann. Mat. Pura Appl. (4) 191 (2012), 219-260.

[16] B. DUCOMET and Š. NEČASOVÁ, Asymptotic behavior of the motion of a viscous heatconducting one-dimensional gas with radiation: the pure scattering case, Anal. Appl. (Singap.) 11 (2013), 29 pp.

[17] B. DUCOMET and Š. NEČASOvÁ, Low Mach number limit in a model of radiative flow, J. Evol. Equ. 14 (2014), 357-385.

[18] S. Eliezer, A. GHATAK and H. HORA, "An Introduction to Equations of States, Theory and Applications", Cambridge University Press, Cambridge, 1986.

[19] E. FeIREISL, "Dynamics of Viscous Compressible Fluids", Oxford University Press, Oxford, 2004.

[20] E. FEIREISL, On the motion of a viscous, compressible, and heat conducting fluid, Indiana Univ. Math. J. 53 (2004), 1707-1740.

[21] E. Feireisl and A. NovotnÝ, "Singular Limits in Thermodynamics of Viscous Fluids", Birkhäuser, Basel, 2009. 
[22] F. GOLSE and B. PERTHAME, Generalized solutions of the radiative transfer equations in a singular case, Comm. Math. Phys. 106 (1986), 211-239.

[23] F. Golse, P.-L. Lions, B. Perthame and R. Sentis, Regularity of the moments of the solution of a transport equation, J. Funct. Anal. 16 (1988), 110-125.

[24] F. Golse, B. Perthame and R. Sentis, Un résultat de compacité pour les équations de transport et application au calcul de la limite de la valeur propre principale d'un opérateur de transport, C. R. Math. Acad. Sci. Paris 301 (1985), 341-344.

[25] D. HofF, Global solutions of the Navier-Stokes equations for multidimensional compressible flow with discontinuous initial data, J. Differential Equations 120 (1995), 215-254.

[26] D. HoFF, Strong convergence to global solutions for multidimensional flows of compressible viscous fluids with polytropic equation of state and discontinuous initial data, Arch. Ration. Mech. Anal. 132 (1995), 1-14.

[27] D. Hoff, Discontinuous solutions of the Navier-Stokes equations for multidimensional flows of heat-conducting fluids, Arch. Ration. Mech. Anal. 139 (1997), 303-354.

[28] N. ITAYA, On the Cauchy problem for the system of fundamental equations describing the movement of compressible viscous fluids, Kodai Math. Sem. Rep. 24 (1971), 60-120.

[29] S. JIANG, Global solutions of the Cauchy problem for a viscous polytropic ideal gas, Ann. Scuola Norm. Sup. Pisa Cl. Sci. (4) 26 (1998), 47-74.

[30] P. JIANG and D. WANG, Global weak solutions to the Euler-Boltzmann equations in radiation hydrodynamics, Quart. Appl. Math. 70 (2012), 25-44.

[31] O. KREML, Š. NEČASOVÁ and M. POKORNÝ, On the steady equations for compressible radiative gas, Z. Angew. Math. Phys. 64 (2013), 539-571.

[32] O.A. LAdYŽEnsKaJA, V.A. SolonNiKov and N.N. URAL'CEVA, "Linear and Quasilinear Equations of Parabolic Type", AMS 1968.

[33] C. LIN, "Mathematical Analysis of Radiative Transfer Models", PhD Thesis, 2007.

[34] C. Lin, J. F. COUlOMBEL and T. Goudon, Shock profiles for non-equilibrium radiative gases, Physica D 218 (2006), 83-94.

[35] C. LIN and T. Goudon, Global existence of the equilibrium diffusion model in radiative model in radiative hydrodynamics, Chin. Ann. Math. 32 B (2011), 549-568.

[36] R. B. LOWrie, J. E. MOREL and J. A. Hittinger, The coupling of radiation and hydrodynamics, Astrophys. J. 521 (1999), 432-450.

[37] A. MATSUMURA and T. NishidA, The initial value problem for the equations of motion of viscous and heat-conductive gases, J. Math. Kyoto Univ. 20 (1980), 67-104.

[38] A. Matsumura and T. NishidA, Initial-boundary value problems for the equations of motion of compressible viscous and heat-conductive fluids, Comm. Math. Phys. 89 (1983), 445-464.

[39] B. Mihalas, "Stellar Atmospheres", W. H. Freeman and Cie, 1978.

[40] B. Mihalas and B. Weibel-Mihalas, "Foundations of Radiation Hydrodynamics", Dover Publications, Dover, 1984.

[41] J. NASH, Le problème de Cauchy pour les équations différentielles d'un fluide général, Bull. Soc. Math. France 90 (1962), 487-497.

[42] G. C. Pomraning, "Radiation Hydrodynamics", Dover Publications, New York, 2005.

[43] J. F. Ripoll, B. Dubroca and G. Duffa, Modelling radiative mean absorption coefficients, Combust. Theory Modelling 5 (2001), 261-274.

[44] V. A. SOLONNIKOV and A. V. KAZHIKOV, Existence theorems for the equations of motion of a compressible viscous fluid, Ann. Rev. Fluid Mech. 13 (1981), 79-95.

[45] E. STEIN, "Singular Integrals and Differentiability Properties of Functions", Princeton University Press, Princeton, New Jersey, 1970.

[46] I. Teleaga and M. SEaïD, Simplified radiative models for low Mach number reactive flows, Appl. Math. Model. 32 (2008), 971-991. 
[47] I. Teleaga, M. Seaïd, I. Gasser, A. Klar and J. Struckmeier, Radiation models for thermal flows at low Mach number, J. Comput. Phys. 215 (2006), 506-525.

[48] X.ZHONG and J. JIANG, Local existence and finite-time blow up in multidimensional radiation hydrodynamics, J. Math. Fluid Mech. 9 (2007), 543-564.

CEA/DAM/DIF

F-91297 Arpajon, France

bernard.ducomet@cea.fr

Institute of Mathematics

of the Academy of Sciences

of the Czech Republic

Žitná 25

11567 Praha 1, Czech Republic

matus@math.cas.cz 